\title{
A NEW TECHNIQUE FOR THE PHOTOSPHERIC DRIVING OF NON-POTENTIAL SOLAR CORONAL MAGNETIC FIELD SIMULATIONS
}

\author{
Marion Weinzierl ${ }^{1}$, Anthony R. Yeates ${ }^{1}$, Duncan H. Mackay ${ }^{2}$, Carl J. Henney ${ }^{3}$, and C. Nick Arge ${ }^{3}$ \\ ${ }^{1}$ Department of Mathematical Sciences, Durham University, South Road, Durham DH1 3LE, UK; marion.weinzierl@durham.ac.uk \\ ${ }^{2}$ School of Mathematics and Statistics, University of St. Andrews, North Haugh, St. Andrews, Fife KY16 9SS, UK \\ ${ }^{3}$ Air Force Research Lab/Space Vehicles Directorate, 3550 Aberdeen Avenue SE, Kirtland AFB, NM, USA \\ Received 2016 February 2; accepted 2016 March 20; published 2016 May 23
}

\begin{abstract}
In this paper, we develop a new technique for driving global non-potential simulations of the Sun's coronal magnetic field solely from sequences of radial magnetic maps of the solar photosphere. A primary challenge to driving such global simulations is that the required horizontal electric field cannot be uniquely determined from such maps. We show that an "inductive" electric field solution similar to that used by previous authors successfully reproduces specific features of the coronal field evolution in both single and multiple bipole simulations. For these cases, the true solution is known because the electric field was generated from a surface flux-transport model. The match for these cases is further improved by including the non-inductive electric field contribution from surface differential rotation. Then, using this reconstruction method for the electric field, we show that a coronal nonpotential simulation can be successfully driven from a sequence of ADAPT maps of the photospheric radial field, without including additional physical observations which are not routinely available.
\end{abstract}

Key words: magnetohydrodynamics (MHD) - Sun: corona - Sun: coronal mass ejections (CMEs) - Sun: magnetic fields

\section{INTRODUCTION}

Non-potential solar coronal magnetic field simulations using the magnetofrictional (MF) method (Yang et al. 1986) have been demonstrated to be a practical alternative to global magnetohydrodynamic (MHD) simulations in long-term computationally expensive coronal studies (see, e.g., Yeates 2014). By allowing for the build up of electric currents and free magnetic energy, the MF method can better approximate physical processes in the corona compared to potential-field source-surface (PFSS) extrapolations. Such non-potential fields have benefits to solar wind and space weather forecasting (Edwards et al. 2015).

Similar to full MHD simulations, global MF simulations of the coronal magnetic field require $E_{\theta}, E_{\phi}$ as inner boundary conditions on the photosphere. In MHD, this horizontal electric field $\boldsymbol{E}_{\perp}$ depends on the vector plasma velocity $\boldsymbol{v}$, the vector magnetic field $\boldsymbol{B}$, and a non-ideal dissipation term. Since complete observations of these quantities are not routinely available, previous authors (see Amari et al. 2003; Fisher et al. 2010; Mackay et al. 2011; Cheung \& DeRosa 2012; Yang et al. 2012; Kazachenko et al. 2014) have shown how this boundary electric field can be inferred from line-of-sight magnetograms to drive both MHD and MF simulations.

In this paper, we present MF simulations. However, the photospheric boundary motions are very slow compared to the coronal Alfvén speed, and the plasma beta is low. As a result, for both MHD simulations and MF simulations, we would expect the coronal magnetic field evolution to closely approximate a sequence of quasi-steady force-free states. Under these approximations, the MHD approach and MF approach produce very similar results. If we are interested primarily in the long-lived structure of the magnetic field rather than the high-frequency dynamics of MHD waves, then the MF method captures the essential evolution more efficiently.

In the case of MF simulations, published results using $\boldsymbol{E}_{\perp}$ determined solely from observed magnetogram sequences have been limited to simulations of individual active regions (Mackay et al. 2011; Cheung \& DeRosa 2012; Gibb et al. 2014). Global simulations, on the other hand, have used specified velocity and dissipation terms to determine $\boldsymbol{E}_{\perp}$ through flux-transport models (Mackay \& Yeates 2012). In this work, for the first time, we explore the driving of global MF simulations directly from sequences of $B_{r}(\theta, \phi, t)$ maps of the whole solar surface, rather than from a flux-transport model. Since we currently have no observed magnetograms of the far side of the Sun, these maps must have been based partly on modeling to determine how $B_{r}$ evolves in the unobserved regions (see Schrijver \& DeRosa 2003; Arge et al. 2010; Henney et al. 2012; Upton \& Hathaway 2014). For this paper, we assume that the sequence of maps has already been constructed.

A particular challenge of applying this technique is the nonuniqueness of $\boldsymbol{E}_{\perp}$ (see Fisher et al. 2010). This is discussed further in Section 4, and essentially reduces to the question of how to approximate the "non-inductive" component of $\boldsymbol{E}_{\perp}$. This is the curl-free component which cannot (in principle) be deduced purely from observations of $\boldsymbol{B}$. In this paper, we illustrate two different approaches for reconstructing $\boldsymbol{E}_{\perp}$ : (i) the non-inductive component is simply neglected (i.e., the simplest solution); (ii) the dominant non-inductive contribution is assumed to come from advection by solar differential rotation, for which the appropriate correction may be computed.

We demonstrate our approaches for estimating $\boldsymbol{E}_{\perp}$ through three different cases. First, we simulate the decay of a single bipolar active region in a low-background magnetic field. Second, we apply our reconstruction approaches to a full Sun $B_{r}(\theta, \phi, t)$ sequence containing multiple evolving bipolar regions. These two sequences of maps are taken from our own surface flux-transport simulations, allowing us to compare the reconstructed electric field with the known electric field from the original simulation. Third, in order to demonstrate the applicability of this method to deal with real observational data, 
we show how our global non-potential coronal magnetic field simulation can be driven by Air Force Data Assimilative Photospheric Flux Transport (ADAPT) maps (Arge et al. 2010; Henney et al. 2012; Hickmann et al. 2015). This poses additional challenges to the method, as new observational data are integrated into these maps when they become available, which produces a time discontinuity that has a non-negligible effect on the simulation (see Section 5.3).

Although we begin with tests based on flux-transport simulations, where $\boldsymbol{E}_{\perp}$ is known, the technique we are developing has much wider applicability. The standard fluxtransport model (Section 3.1) has three main components: two advection velocities (meridional flow and differential rotation) and a macroscopic diffusion term. While advection acts over all spatial scales, the diffusion is designed to represent the net effect of the random walk of many small convective cells dispersing magnetic flux over the solar surface, thus limiting the standard flux-transport model to spatial scales larger than that of the supergranulation. Under these approximations, the flux-transport model successfully models the evolution of the global magnetic field and the dispersal of flux out of active regions. It cannot, however, model the complex small-scale dynamics that occur within active regions. Nor can it account for localized variations in the solar surface flows. We aim to circumvent these limitations by developing a technique to drive MF simulations from arbitrary sequences of magnetic maps. Although we begin by validating against the standard fluxtransport model, future simulations will be limited only by the spatial resolution of observations and the available computing power.

This paper is organized as follows. The non-potential coronal model we use is described in Section 2, and the three cases of simulated input $B_{r}$ maps are described in Section 3. Our methods for electric field inversion are described in Section 4, and the resulting non-potential simulations are discussed in Section 5.

\section{NON-POTENTIAL CORONAL MODEL}

The magnetofrictional (MF) technique follows the evolution of the large-scale magnetic field in the solar corona. Instead of solving the full MHD momentum equation, the velocity $\boldsymbol{v}$ is approximated by the magnetofrictional form introduced by Yang et al. (1986), namely,

$$
\boldsymbol{v}=\nu^{-1}\left(\boldsymbol{J} \times \boldsymbol{B} / B^{2}\right),
$$

where $\boldsymbol{J}=\nabla \times \boldsymbol{B}$ and $\nu$ is a friction coefficient. This form enforces the relaxation of the magnetic field toward a nonlinear force-free state where $\boldsymbol{J} \times \boldsymbol{B}=0$. The MF model allows for the gradual build up and conservation of magnetic energy and electric currents in the corona, in contrast to common extrapolation methods, which have no memory of magnetic connectivity.

The two flowcharts in Figure 1 indicate how the nonpotential magnetic field is evolved over time, contrasting previous flux-transport driven simulations (Figure 1(a)) with the new simulations described in this paper (Figure 1(b)). In both cases, the vector potential $\boldsymbol{A}$ in the coronal volume is evolved through the same MF technique, and the time stepping is done using the Euler method. The difference lies in how the lower boundary condition for $\boldsymbol{E}_{\perp}$ is implemented. In the original simulations (Figure 1(a); e.g., Yeates et al. 2010), $\boldsymbol{E}_{\perp}$ is given by a surface flux-transport model with imposed flows, whereas, in the new simulations (Figure 1(b)), $\boldsymbol{E}_{\perp}$ is determined directly from a sequence of surface magnetic maps. For the single and multiple bipole simulations, the hyperdiffusion coefficient is set to zero, whereas, in the simulations using the ADAPT maps, it is $1 \times 10^{11} \mathrm{~km}^{4} \mathrm{~s}^{-1}$.

All of the simulations in this paper use a fixed, regular spherical grid $R_{\odot} \leqslant r \leqslant 2.5 R_{\odot}, 8.3 \leqslant \theta \leqslant 171^{\circ} .7$ (i.e., the poles are cut off), $0^{\circ} \leqslant \phi \leqslant 360^{\circ}$, with grid cells equally spaced in the stretched coordinates $x, y, z$ (see Appendix A.1), with 28 cells in radius, 160 cells in latitude, and 192 cells in longitude. At the latitudinal boundaries $\left(\theta=8^{\circ} .3\right.$ and $\theta=171^{\circ} .7$ ), we impose $B_{\theta}=v_{\theta}=0$. The inner boundary conditions (at $r=R_{\odot}$ ) are the main subject of this paper (see Section 4$)$. At the outer boundary $\left(r=2.5 R_{\odot}\right)$, we follow our previous simulations and impose zero radial gradient in $B_{\theta}$ and $B_{\phi}$, along with a radial outflow to ensure that $\boldsymbol{B}$ remains predominantly radial near this boundary, while allowing flux ropes to be ejected (see Mackay \& van Ballegooijen 2006; Yeates et al. 2010).

\section{INPUT DATA}

In this paper, we consider three different sequences of $B_{r}(\theta, \phi, t)$ for the photospheric boundary conditions, with corresponding initial conditions for $\boldsymbol{B}(r, \theta, \phi, \boldsymbol{t}=\boldsymbol{0})$. Two sequences are taken from our own surface flux-transport simulations, while the third is taken from the ADAPT model. From first to third, the test sequences have decreasing amounts of available information about $\boldsymbol{E}_{\perp}$, in addition to the sequence of $B_{r}$ itself. Using test cases where $\boldsymbol{E}_{\perp}$ is known allows us to validate our reconstructed $\boldsymbol{E}_{\perp}$, as well as to compare threedimensional (3D) output quantities such as photospheric flux, open flux, magnetic energy, or electric current. In principle, any data processed to give a $360^{\circ}$ radial magnetic map of the Sun can be used as input to our method. However, we will see in Section 5.3 that the quality of the map (for example, how new data is assimilated) has an impact on the outcome. As the method is not limited to larger spatial scales, future simulations (given sufficient computing resources) could model local regions of the Sun directly from observed magnetograms at their full resolution. An application of a similar technique to active regions observed by Solar and Heliospheric Observatory/MDI can be seen in Gibb et al. (2014).

\subsection{Single-bipole Simulation}

For this sequence, a single bipolar magnetic region is evolved for over $1200 \mathrm{hr}$ (50 days), under the influence of surface flux-transport effects, but with no flux emergence during the simulation (see Mackay \& van Ballegooijen 2001, 2005, 2006). Here, the sequence of $B_{r}(\theta, \phi, t)$ maps is computed from the surface flux-transport model, whose electric field $\boldsymbol{E}_{\perp}(\theta, \phi, t)$ is known completely and given by

$$
\boldsymbol{E}_{\perp}=-\boldsymbol{v} \times\left(B_{r} \boldsymbol{e}_{r}\right)+D \nabla \times\left(B_{r} \boldsymbol{e}_{r}\right),
$$

where $D=500 \mathrm{~km}^{2} \mathrm{~s}^{-1}$ is the supergranular diffusivity and $v$ is a steady axisymmetric flow comprising a poleward meridional flow $u_{\theta}$ (due to Schüssler \& Baumann 2006) along with differential rotation $u_{\phi}$ (Snodgrass 1983), expressed in the 


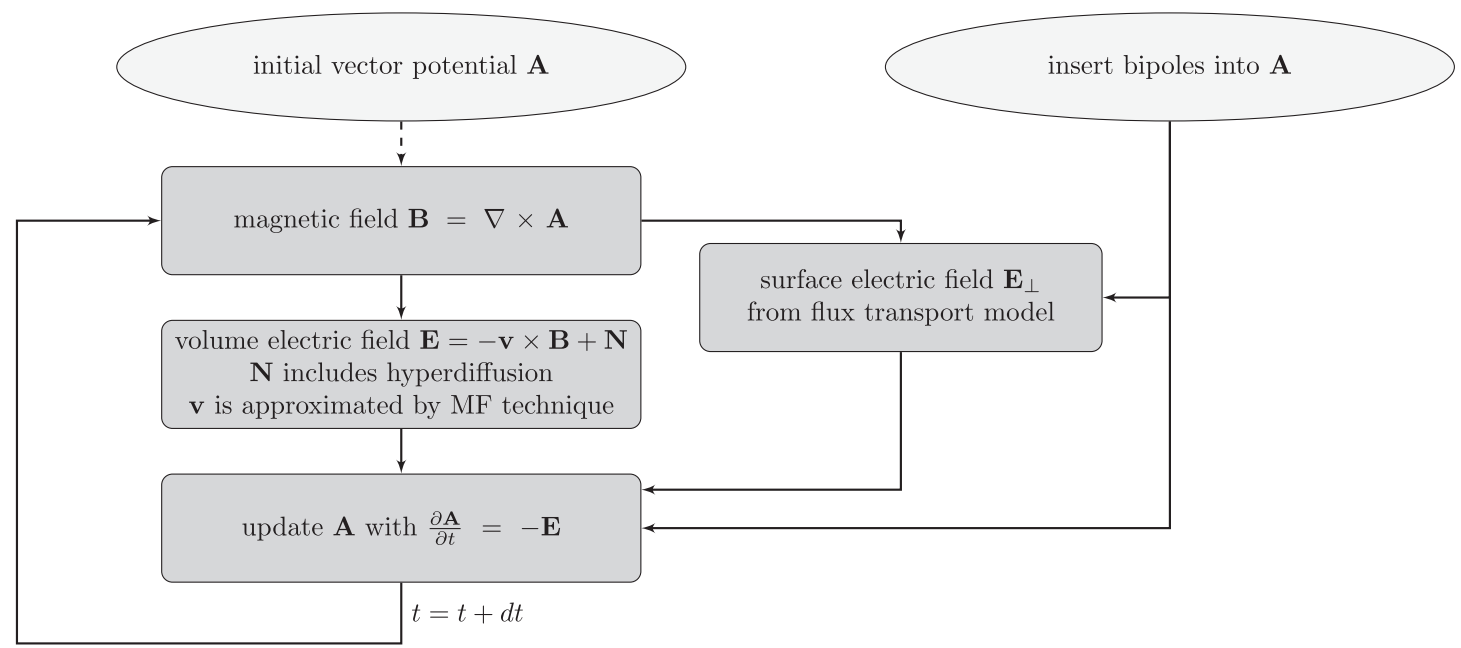

(a) The evolution of the magnetic field in the original MF simulation.
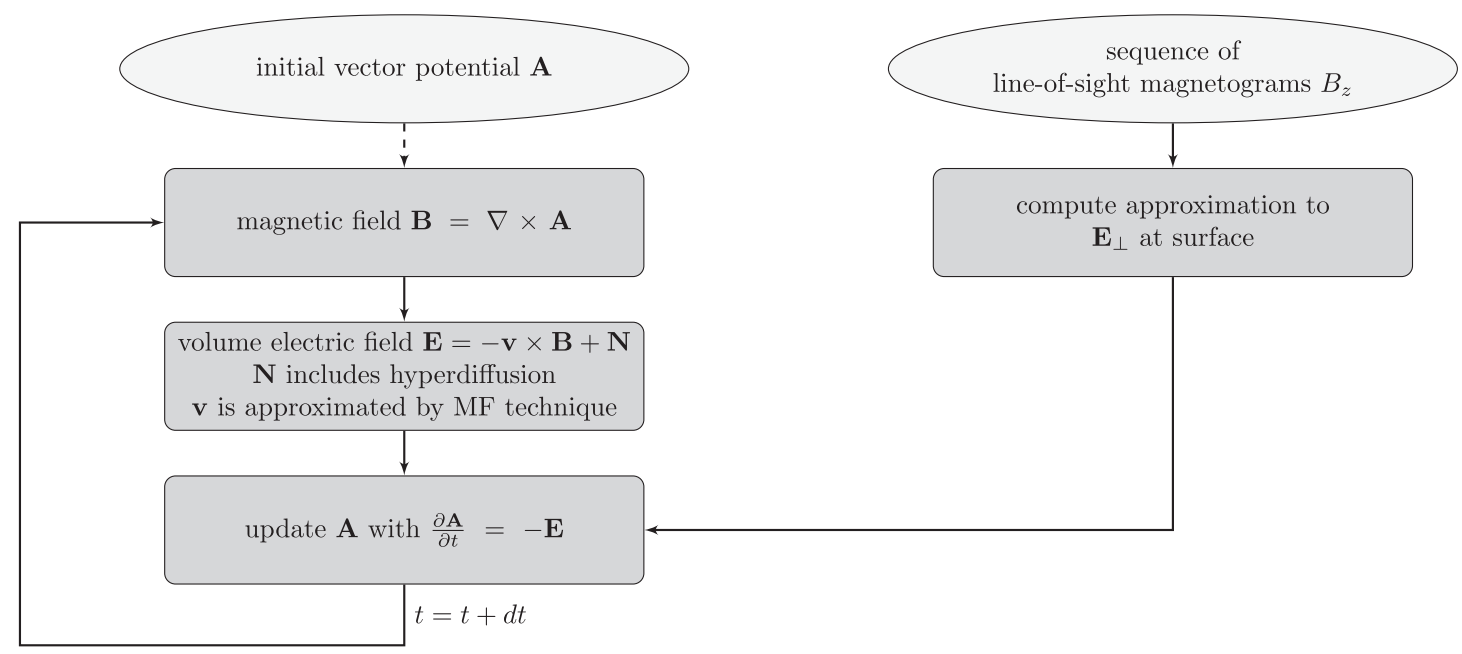

(b) The evolution of the magnetic field in the MF simulation using magnetograms on the photospheric boundary to drive the simulation.

Figure 1. Simulation flowcharts.

Carrington frame, given by

$$
\begin{gathered}
u_{\theta}=u_{0} \frac{16}{110} \sin (2 \lambda) \exp (\pi-2|\lambda|), \\
u_{\phi}=\Omega(\phi) R_{\odot} \sin \theta, \\
\Omega=0.18-2.3 \cos ^{2} \theta-1.62 \cos ^{4} \mathrm{deg}_{\mathrm{day}}{ }^{-1},
\end{gathered}
$$

with $\lambda=\pi / 2-\theta$ and $u_{0}$ being a constant controlling the flow amplitude. The $B_{r}(\theta, \phi, t)$ map is saved once per hour in order to test our reconstruction of $\boldsymbol{E}_{\perp}$.

The initial magnetic bipole takes the form given by Mackay \& van Ballegooijen (2001), which also defines its initial 3D magnetic field for the MF simulations. This initial 3D magnetic field is not potential, but includes a non-zero twist in the center of the bipole. For this test, the bipole is embedded in a weak background magnetic field, computed by assuming a potential field with $B_{r}\left(R_{\odot}, \theta, \phi\right)=0.01 \cos ^{7} \theta$.

\subsection{Multiple-bipole Simulation}

The second sequence of $B_{r}(\theta, \phi, t)$ is taken from the same surface flux-transport model as the single bipole simulation. The main difference is that now multiple bipolar regions emerge during a continuous evolution over $1512 \mathrm{hr}$ (63 days). This represents the photospheric driver used in the global MF simulations that have been carried out to date by Yeates et al. (2008, 2010), Yeates \& Mackay (2012), and Yeates (2014). The simulation is initialized with a potential-field extrapolation from an observed synoptic magnetogram (corrected for differential rotation). The simulated time is 2012 September 11, 12:00 UT, to 2012 November 13, 12:00 UT.

In this case, $\boldsymbol{E}_{\perp}$ still follows the known evolution described by flux-transport processes (2) between emergence events. However, the new bipoles emerge instantaneously (always at 12:00 on the corresponding day), following the method described by Yeates et al. (2008). The $B_{r}(\theta, \phi, t)$ sequence is therefore discontinuous in time, with each bipole emergence corresponding to an instantaneous impulse of $\boldsymbol{E}_{\perp}$ of infinite 
strength. We will show in Section 5.2 how our method of reconstructing $\boldsymbol{E}_{\perp}$ approximates this impulse with an electric field of finite strength and duration, depending on the chosen cadence between maps in the $B_{r}$ sequence.

\subsection{ADAPT Maps}

Our third sequence of $B_{r}(\theta, \phi, t)$ is taken from the the global magnetic maps generated by the ADAPT model. ADAPT uses a photospheric flux-transport model based on that of Worden \& Harvey (2000), together with data assimilation techniques (in this case, ensemble least squares) from the Los Alamos National Laboratory (LANL) framework, in order to generate more realistic global photospheric magnetic field maps. The flux-transport model includes differential rotation and meridional flow similar to the model used in Sections 3.1 and 3.2. However, instead of a simple diffusion model of supergranulation, it models the small-scale clumping of magnetic flux on the solar surface through a combination of random attractors and random daily background flux emergence.

Magnetograms from the National Solar Observatory (NSO) Global Oscillation Network Group (GONG; Harvey et al. 1996) are used as the input data source to produce the ADAPT maps employed in this work. The ADAPT model assimilates new observations once per day from line-of-sight magnetograms, or, if no new observations are available (due to the weather), from the evolved model. Between the daily data assimilation steps, maps of $B_{r}(\theta, \phi, t)$ are output every two hours from the model, and it is these maps that we use as input. The sequence of maps used here runs from 2014 November 1 , 00:00 UT, to 2014 December 23, 22:00 UT (1270 hr). None of the maps we use correspond exactly to the time of data assimilation.

While the data assimilation ensures that Earth-facing regions of the solar surface match more closely to observations, it does mean that the true $\boldsymbol{E}_{\perp}$ is not known for the ADAPT sequence. This is in contrast to the bipole simulations where it is known. As a result, it is important to begin by testing the electric field inversion on known cases of the bipole sequences before applying it to the ADAPT sequence.

As only half of the Sun is visible from the Earth at one time, the far-side data in the ADAPT maps are outdated, using only previous observations and the flux-transport model. The data for these regions are only updated as the regions appear again on the east limb, with the data assimilation technique taking into account the observational errors at the limb. This, however, creates a discontinuity in the data at the respective longitude, particularly when significant flux emergence or active-region evolution has taken place on the far side. This has some effect on the simulation, as we will see in Section 5.3.

Before computing $\boldsymbol{E}_{\perp}$, we rotate the ADAPT maps into the Carrington frame, interpolate them to match our computational grid (from an original resolution of $360 \times 180$ ), and remove any monopole component using a multiplicative flux correction. The ADAPT maps come as an ensemble of 12 realizations which account for model parameter uncertainties in the supergranular flow. We arbitrarily picked one of these realizations for our runs in Section 5.3, although we have verified that the results are only slightly changed if a different realization is selected.

\section{METHODS FOR ELECTRIC FIELD INVERSION}

The basic problem is to determine $\boldsymbol{E}_{\perp}$ from $B_{r}$, where the two quantities are related through the radial component of Faraday's law:

$$
\frac{\partial B_{r}}{\partial t}=-\boldsymbol{e}_{r} \cdot \nabla \times \boldsymbol{E}_{\perp}
$$

It is convenient to make a Helmholtz decomposition of $\boldsymbol{E}_{\perp}$ (e.g., Amari et al. 2003; Fisher et al. 2010) and express it as

$$
\boldsymbol{E}_{\perp}=-\nabla \times\left(\frac{\partial \Phi}{\partial t} \boldsymbol{e}_{r}\right)-\nabla_{\perp} \frac{\partial \psi}{\partial t},
$$

where the time derivatives are included for later convenience. As we will show below, the potential $\Phi(\theta, \phi, t)$ may be determined from a sequence of $B_{r}$ maps using Equation (5) (see also Fisher et al. 2010; Cheung \& DeRosa 2012; Yang et al. 2012). For this reason, we call the first part of the decomposition the "inductive component."

In contrast, the potential $\psi(\theta, \phi, t)$ is impossible to determine from $B_{r}$ using Equation (5), since the horizontal gradient vanishes under the operation $\boldsymbol{e}_{r} \cdot \nabla \times$. We call this the "non-inductive" component. This freedom means that the total electric field $\boldsymbol{E}_{\perp}$ is not uniquely determined by $B_{r}$ alone. The simplest practical solution is to ignore the non-inductive component (i.e., set $\psi=0$ ), as in the works of Mackay et al. (2011), Yang et al. (2012), and Gibb et al. (2014). However, we will show that this component is non-negligible in our bipole simulations, leading to significant differences in the coronal magnetic field. We will therefore propose, in Section 4.2, a practical first-order approximation to this component for global simulations.

\subsection{Purely Inductive Solution}

For a given sequence $B_{r}(\theta, \phi, t)$, the inductive component $-\nabla \times\left(\partial \Phi / \partial t \boldsymbol{e}_{r}\right)$ is uniquely determined for suitable boundary conditions. To appreciate the solution uniqueness, we substitute Equation (6) into Faraday's law (5) to obtain the twodimensional Poisson equation

$$
\nabla_{\perp}^{2} \frac{\partial \Phi}{\partial t}=-\frac{\partial B_{r}}{\partial t} .
$$

In practice, we follow Mackay et al. (2011) in the equivalent method of first solving the Poisson equation

$$
\nabla_{\perp}^{2} \Phi=-B_{r}
$$

then taking the time derivative. On the full sphere, this equation would have a unique solution for $\Phi$. However, our simulation domain omits the poles, and so it is necessary to impose boundary conditions on the $\theta$ boundaries. As a physically reasonable choice, we take the Neumann boundary conditions $\partial \Phi / \partial \theta=0$, so that $E_{\phi}=0$ on these boundaries, which is consistent with global magnetic flux conservation. To solve the Poisson equation, we have implemented a spherical multigrid solver (see Appendix A.2).

The purely inductive approximation

$$
\boldsymbol{E}_{\perp}=-\nabla \times\left(\frac{\partial \Phi}{\partial t} \boldsymbol{e}_{r}\right)
$$




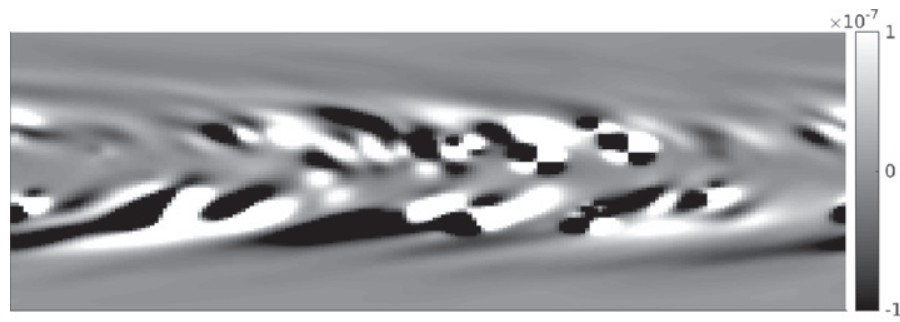

(a) $E_{\phi}$ original multiple bipole simulation

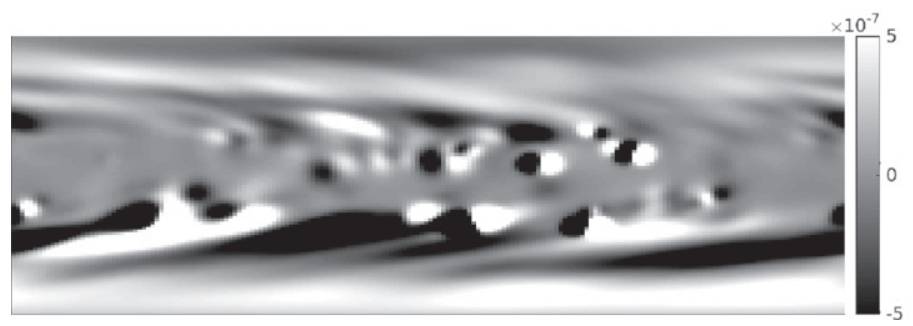

(c) $E_{\theta}$ original multiple bipole simulation.

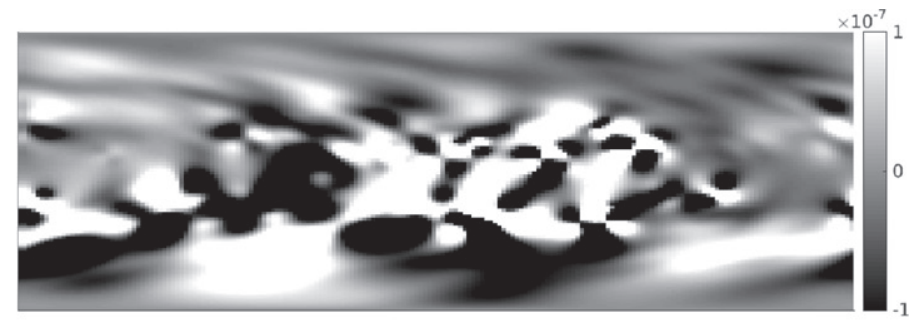

(b) $E_{\phi}$ inductive-only reconstruction.

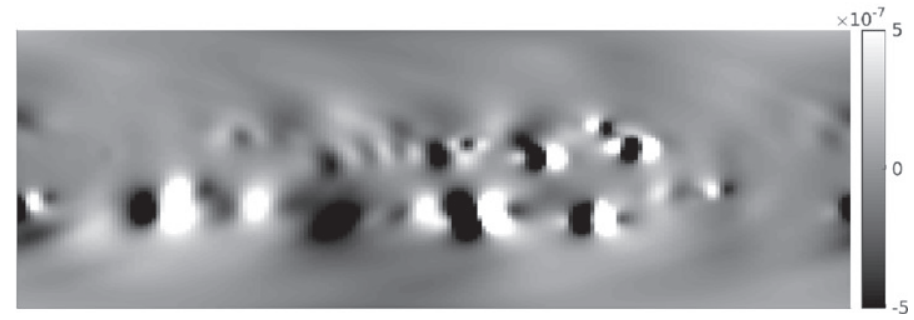

(d) $E_{\theta}$ inductive-only reconstruction.

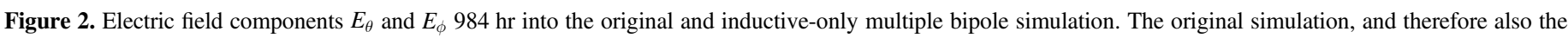
input maps for the data-driven simulation, include differential rotation.

is, computationally speaking, the simplest electric field consistent with a given $B_{r}$ sequence. To illustrate that in the global simulations this term alone is not satisfactory, Figure 2 shows a snapshot of the purely inductive $\boldsymbol{E}_{\perp}$ components $E_{\theta}, E_{\phi}$ derived from the multiple bipole sequence, alongside the true electric field components (from the flux-transport simulations). It can be clearly seen, particularly at high latitudes, that the inductive-only version neglects to include the east-west structures (most obviously for $E_{\theta}$ ) which are a consequence of differential rotation. It is not surprising that the inductive component misses this as at higher latitudes the field becomes more axisymmetric. As a consequence, differential rotation has the effect of acting along contours of the same value of $B_{r}$. Thus, when the time derivative of Equation (8) is taken, no change is found. The next section describes our approach to improve the approximation to $\boldsymbol{E}_{\perp}$; further comparisons will be given in Section 5.

\subsection{Non-inductive Component}

Fisher et al. (2012) and Kazachenko et al. (2014) use additional observations (e.g., Doppler velocities) and assumptions (such as ideal Ohm's law) in order to estimate the noninductive component $-\nabla_{\perp} \partial \psi / \partial t$ in individual active regions. These additional measurements, however, are not routinely available for the full solar surface including the far side.

Here, we propose an alternative way to approximate the noninductive component, using only routinely available global line-of-sight magnetograms and knowledge of large-scale differential rotation. Our approach is motivated by the observation that for the multiple bipole sequence, the purely inductive approximation works well for a sequence where differential rotation is turned off. This is shown in Figure 3 where both $E_{\theta}$ and $E_{\phi}$ show much better agreement than in the original Figure 2.

Since we know the differential rotation velocity $\boldsymbol{v}=v_{\phi}(\theta) \boldsymbol{e}_{\phi}$ that produced the multiple bipole sequence, we can include the corresponding non-inductive component from the ideal Ohm's law $\boldsymbol{E}_{\perp}=-\boldsymbol{v} \times\left(B_{r} \boldsymbol{e}_{r}\right)$. Taking the divergence removes the inductive component, leaving a new Poisson equation:

$$
\nabla_{\perp}^{2} \frac{\partial \psi}{\partial t}=\nabla \cdot\left(v_{\phi} B_{r} \boldsymbol{e}_{\theta}\right),
$$

which we solve to provide an approximation to $\partial \psi / \partial t$. For consistency with the the inductive part, we have to impose homogenous Dirichlet boundary conditions (i.e., $\psi=0$ ) on the $\theta$ boundary. Since in global simulations differential rotation is the dominant large-scale flux-transport effect with a timescale of $1 / 4$ year, we expect that this will be the dominant contribution to the non-inductive electric field, at least away from times when new bipoles emerge. The correction may also be applied to the ADAPT sequence, which we show produces reasonable results, although we will see that the purely inductive solution actually works rather well in that case.

Figure 4 shows that the reconstructed electric field components, which include the non-inductive component, are in better agreement with the original multiple bipole sequence. In the following section, Section 5, we discuss the effect of the reconstructed non-inductive component in more detail for the simulations with different input sequences.

\section{RESULTS}

\subsection{Single-bipole Sequence}

The comparisons in Figures 2-4 suggest that including a non-inductive component in the reconstruction of $\boldsymbol{E}_{\perp}$ can improve the match to the original flux-transport $\boldsymbol{E}_{\perp}$, at least away from times of flux emergence. To quantify this effect more carefully, we begin by examining 3D simulation results for the simplest case of a single bipole time sequence.

Figure 5 shows four diagnostic quantities for the nonpotential simulations. The different curves show a simulation driven by the original $\boldsymbol{E}_{\perp}$ (green), one driven by the purely inductive $\boldsymbol{E}_{\perp}$ (red), and one driven by the combined ("improved") $\boldsymbol{E}_{\perp}$ (i.e., including our approximation of the non-inductive component, blue line). While the photospheric 


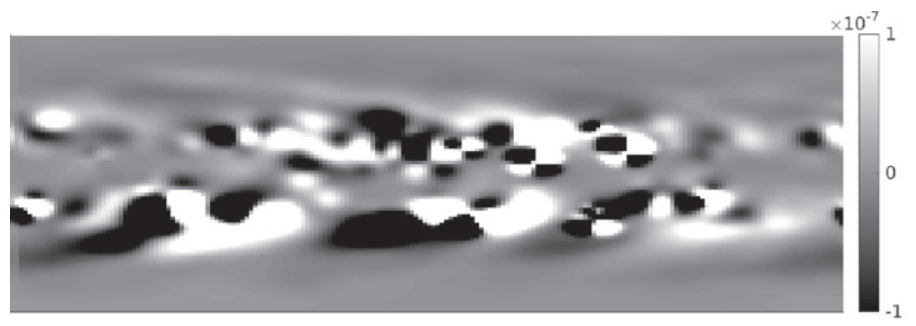

(a) $E_{\phi}$ original multiple bipole simulation.

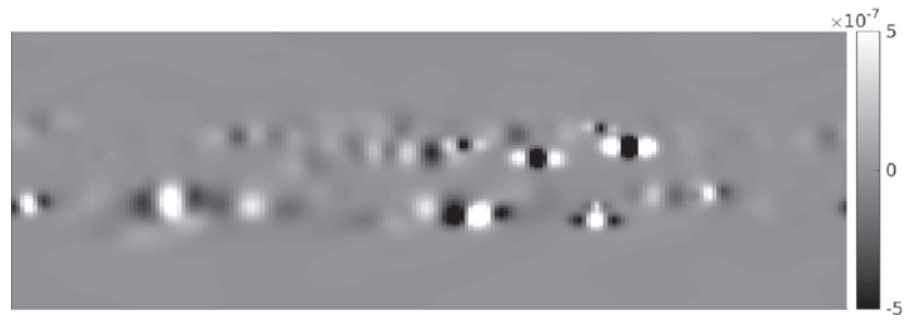

(c) $E_{\theta}$ original multiple bipole simulation.

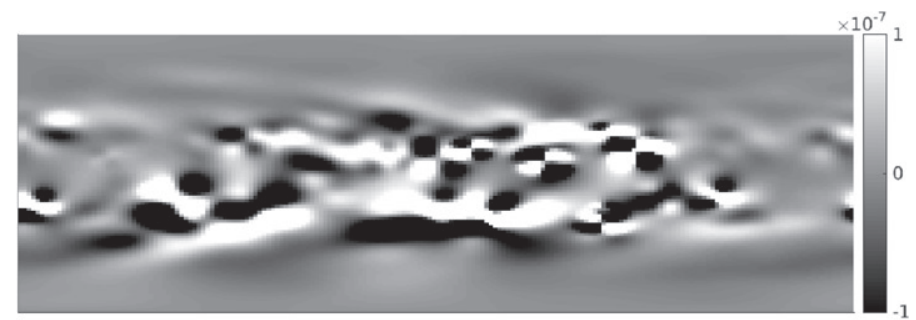

(b) $E_{\phi}$ inductive-only reconstruction.

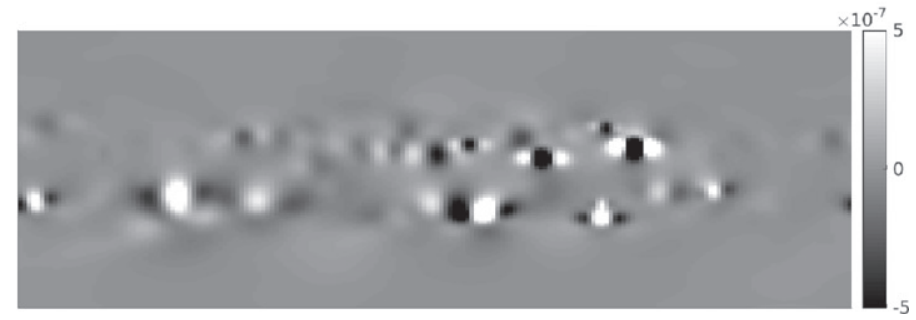

(d) $E_{\theta}$ inductive-only reconstruction.

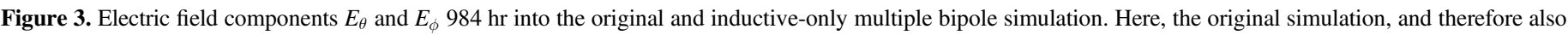
the input maps for the data-driven simulation, do not include differential rotation.

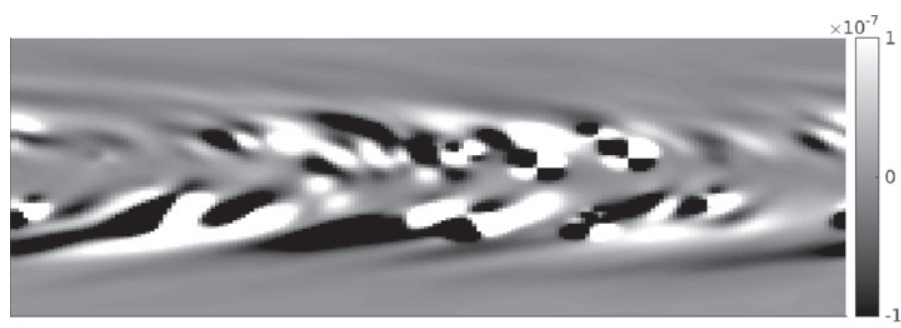

(a) $E_{\phi}$ original multiple bipole simulation.

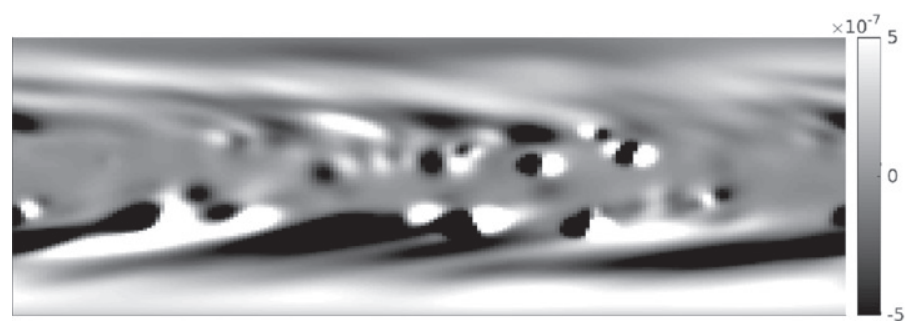

(c) $E_{\theta}$ original multiple bipole simulation.

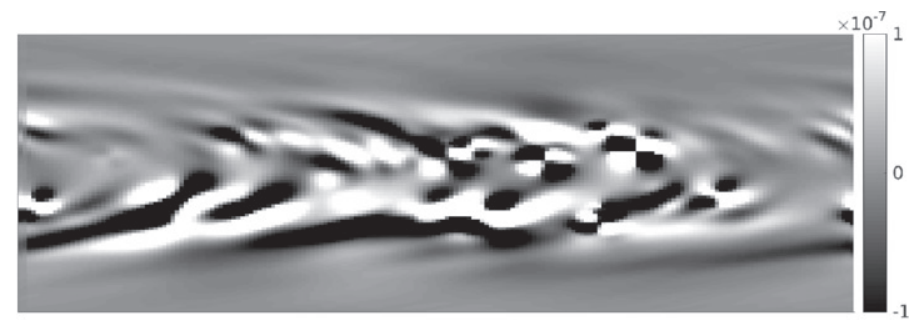

(b) $E_{\phi}$ improved reconstruction.

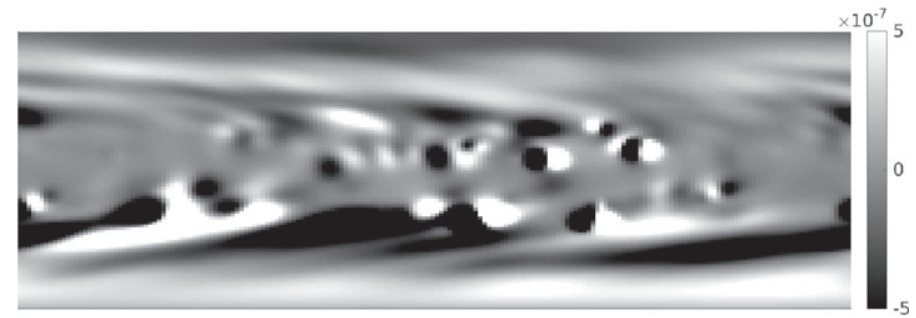

(d) $E_{\theta}$ improved reconstruction.

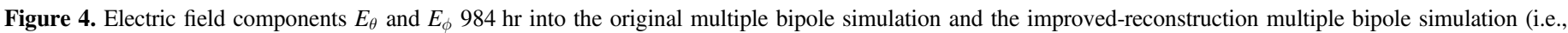
including the non-inductive part). The original simulation, and therefore also the input maps for the data-driven simulation, include differential rotation.

flux is the same in all of the cases (since all three $\boldsymbol{E}_{\perp}$ produce the same $B_{r}$ ), the benefit of the combined reconstruction becomes clear from the three coronal diagnostics. The most significant feature of this particular MF simulation is the gradual formation and strengthening of a flux rope above the bipole center (see Mackay \& van Ballegooijen 2006 and Mackay 2006 for a similar case study), followed by its sudden eruption (after about $670 \mathrm{hr}$ in the original simulation). The rise and ejection of this flux rope is most obviously seen in the open flux. While both reconstructions lead to an eruption, only the combined reconstruction reproduces the original simulation timing. The timing of the ejection is delayed by almost $500 \mathrm{hr}$ in the purely inductive simulation. The combined solution follows the original simulation closely, with only a short delay in the eruption. It is also clear that for the magnetic energy and electric current, the combined simulation produces a much more realistic reproduction of the original simulation. This indicates that differential rotation, which is included through the non-inductive term, plays a key role in the evolution of the coronal field.

Figure 6 exhibits model snapshots of the magnetic field lines, traced from identical starting points, before and after the original flux rope eruption. The bipole can be seen in black and white on the gray sphere. The three cases are compared in Figure 6. For example, after $240 \mathrm{hr}$ (shown in the first row), the shape of the field lines is still quite similar in all three cases, however, by $600 \mathrm{hr}$ (middle row) the original (green) and improved-reconstruction (blue) plots show a strongly sheared 

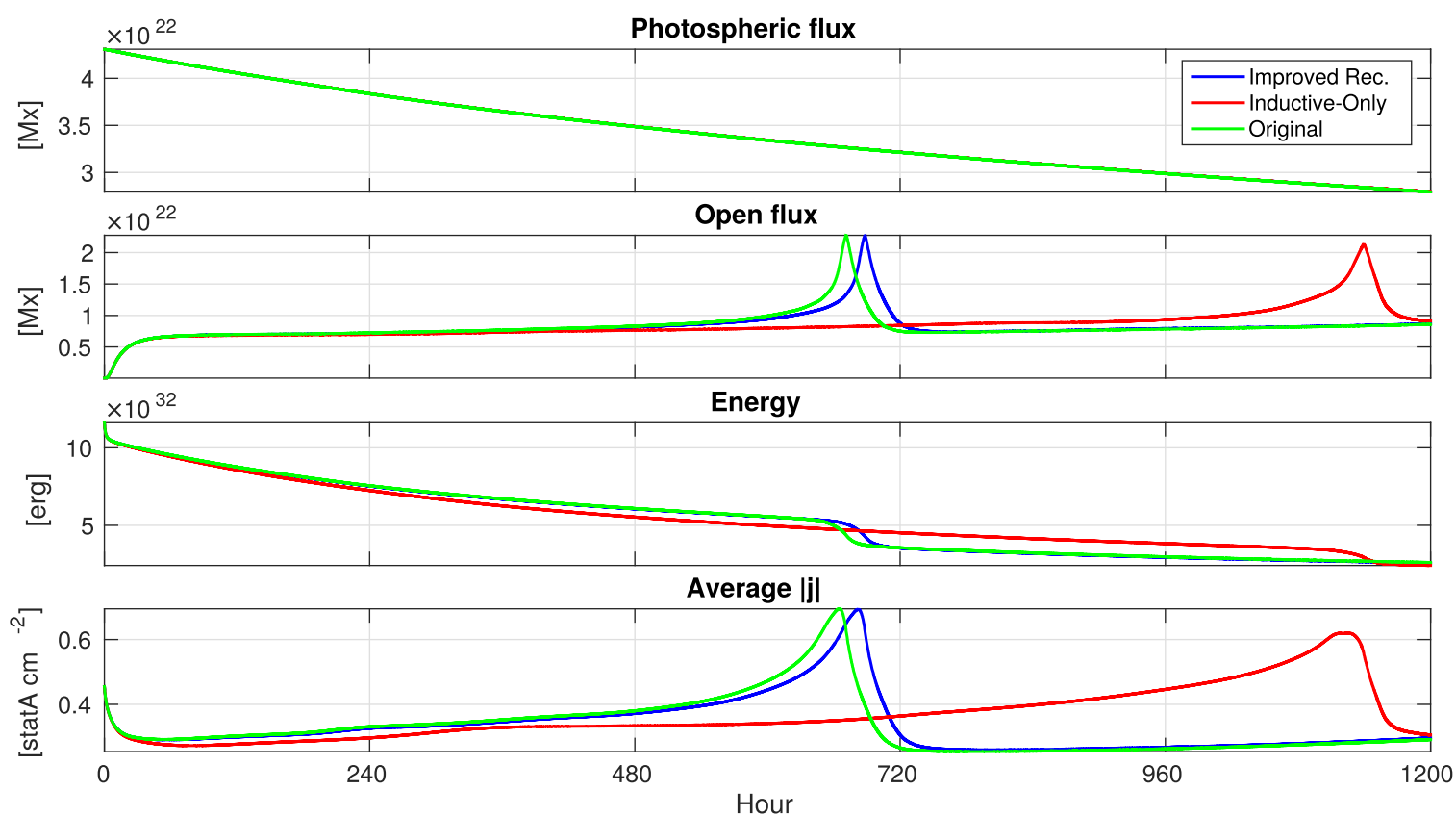

Figure 5. Integrated quantities in the single bipole simulations. The top panel shows the total unsigned magnetic flux through the photosphere, the second panel represents the total unsigned open magnetic flux (i.e., through the outer boundary), the third panel depicts the total magnetic energy, and the fourth panel features the averaged electric current $|j|$ in the volume.

field over the polarity inversion line (PIL). This strongly sheared field is not present for the inductive-only plot (red). In the bottom row (hour 696), the original field lines have already almost settled down again after the eruption and the combinedreconstruction field lines have just erupted, whereas the inductive-only field lines are still in the process of building up a sheared field. Overall, the structure and shape of the blue and green field lines are very similar.

To quantify the amount of sheared field built up above the PIL, Yeates \& Mackay (2012) defined a measure called "skew." This is the sine of the angle between the horizontal field $\boldsymbol{B}_{\perp}$ at a certain height $r>R_{\odot}$ and the direction $\nabla B_{r}$ (normal to the PIL) in the photosphere, i.e.,

$$
\sin \gamma=\frac{\boldsymbol{e}_{r} \cdot \nabla B_{r} \times \boldsymbol{B}_{\perp}}{\left|B_{r}\right|\left|\boldsymbol{B}_{\perp}\right|}
$$

A magnitude of $|\sin \gamma|=1$ indicates that the field at height $r$ is directed along the PIL, while $\sin \gamma=0$ indicates that the field is perpendicular to the PIL. Here, we use $\sin \gamma$ to compare the amount of horizontal magnetic field built up in the corona by the different reconstructions of $\boldsymbol{E}_{\perp}$. Figure 7(a) shows the maximum skew (along the length of the PIL) at a height of $r=0.3448 R_{\odot}$ for the three cases. The skew increases until the flux rope erupts in each case, and then declines rapidly. Again, the simulation driven by the combined reconstruction matches the original simulation closely in this measure, while the build up of skew is significantly slower in the purely inductive case. Clearly, the non-inductive electric field from differential rotation makes an important contribution here.

An alternative comparison between the inductive and combined reconstructions is given by Figure 7(b), which shows the correlation for $B_{\theta}$ ( $B_{\phi}$ gives similar results) between each simulation and the original (averaged over $r$ ). The correlation is almost perfect for the improved reconstruction, except for a dip around the time of the flux rope eruption. This corresponds to the slight delay in the eruption compared to the original simulation, which is likely due to the omitted contribution of supergranular diffusion to the non-inductive electric field. For the purely inductive simulation, the correlation is significantly worse at all times.

\subsection{Multiple-bipole Sequence}

We have already seen in Figures 2 and 4 how including the non-inductive component improves the agreement of our simulated electric field with the original field from the multiple bipole simulation. Figure 8 shows how other simulated quantities, including magnetic flux, magnetic energy, and electric current, differ when we omit or include the noninductive component in the multiple bipole simulation. For the original simulation (green line), one can see the jumps in energy and photospheric flux, and the corresponding drops in the open flux, when new bipoles are inserted into the simulation domain. These drops are not visible in the two reconstructed versions, as they are due to the idealized bipole insertion procedure of the original simulation and vanish when we interpolate the electric field between the times when new $B_{r}$ maps are imposed. The photospheric flux matches closely, as it is imposed each time a new map is incorporated for both reconstruction cases. The total electric current and the magnetic energy match quite closely for both cases, although the inductive-only simulation leads to a slightly higher average current. The difference in the open flux becomes larger over time, with more open flux and a bigger difference compared to the original simulation in the inductive-only case.

The plots in Figure 9 show the progression of the correlation coefficient values with time between $B_{\theta}$ of the two data-driven runs and that of the original simulation. The correlation is initially very good, but decreases with time. It is clear that the improved reconstruction produces much better results, with its 


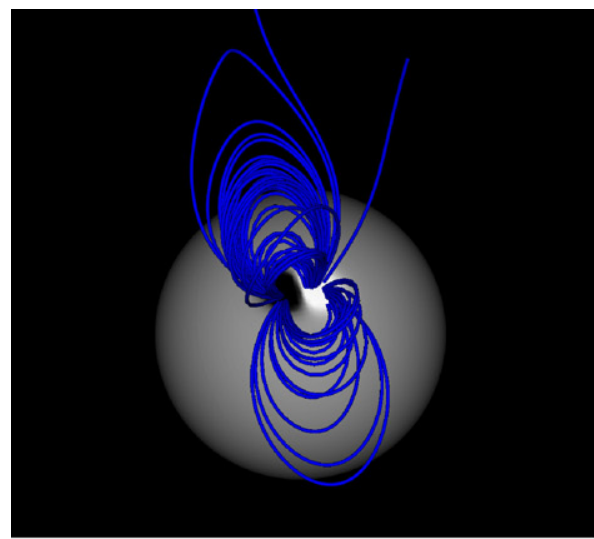

(a) Improved, hour 240

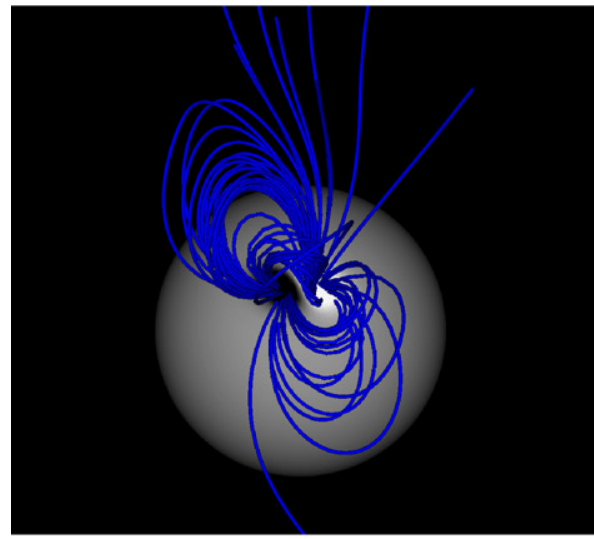

(d) Improved, hour 600

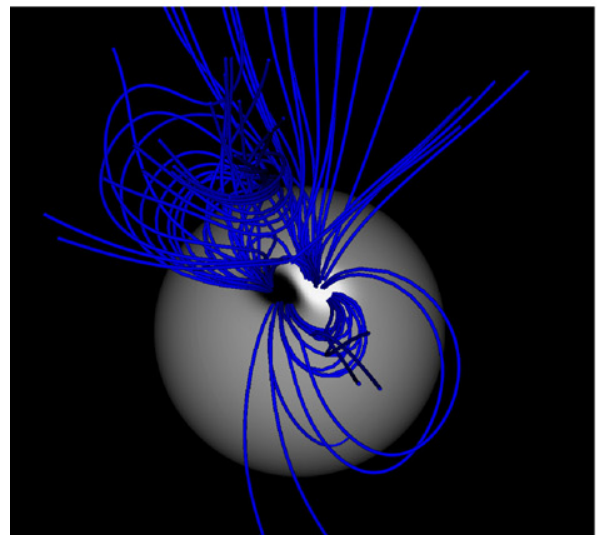

(g) Improved, hour 696

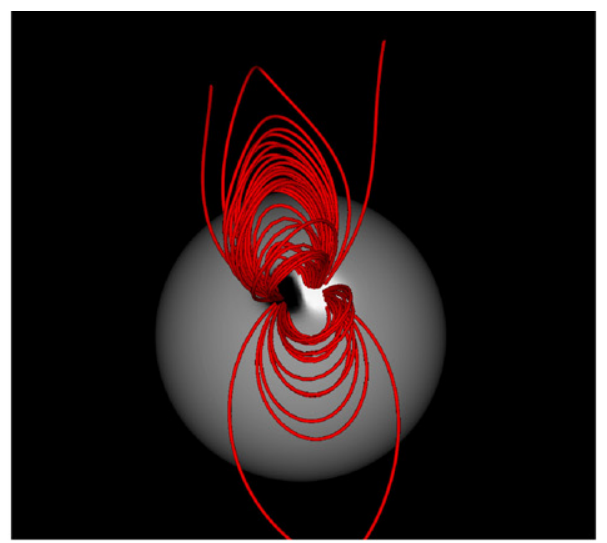

(b) Inductive-only, hour 240

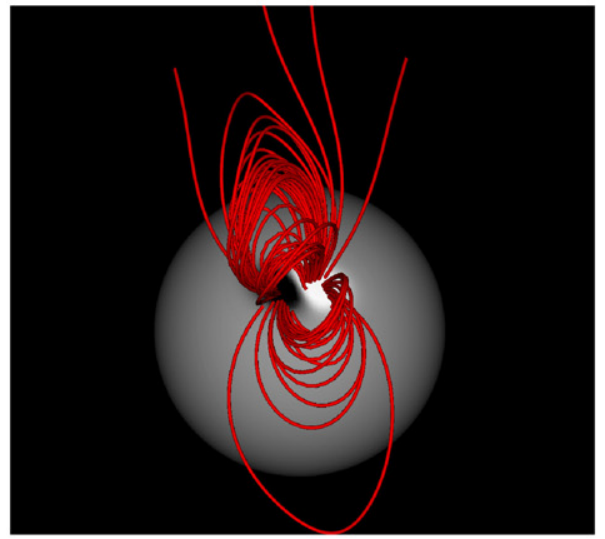

(e) Inductive-only, hour 600

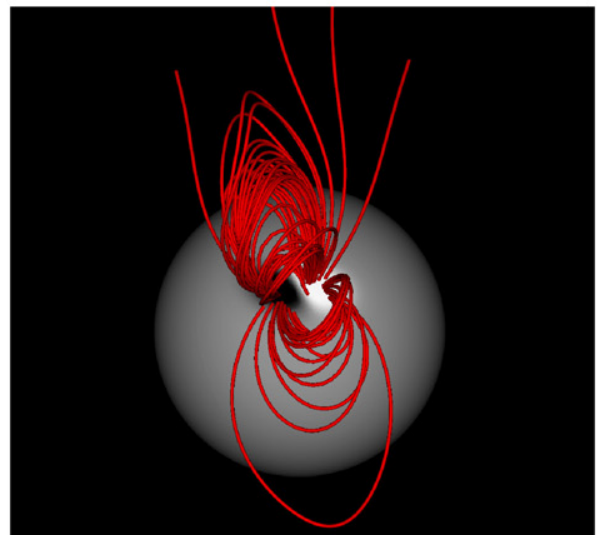

(h) Inductive-only, hour 696

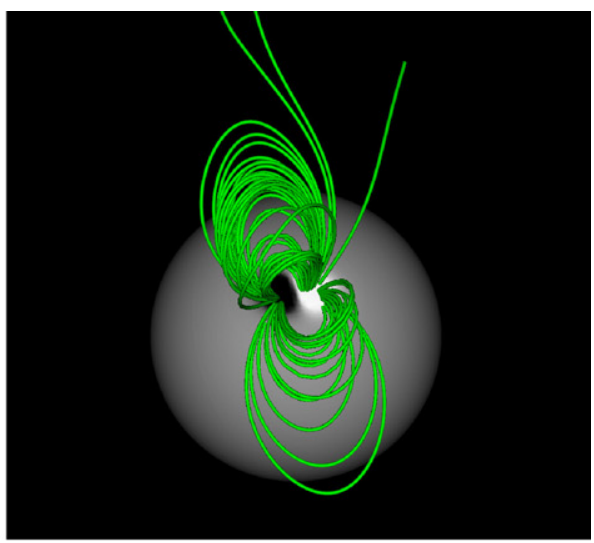

(c) Original, hour 240

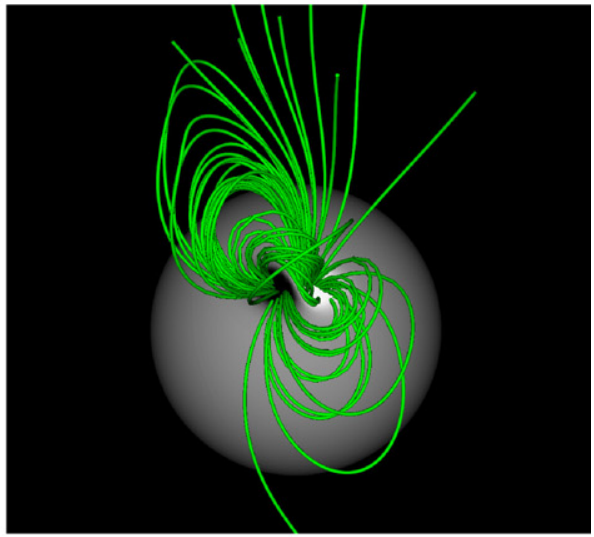

(f) Original, hour 600

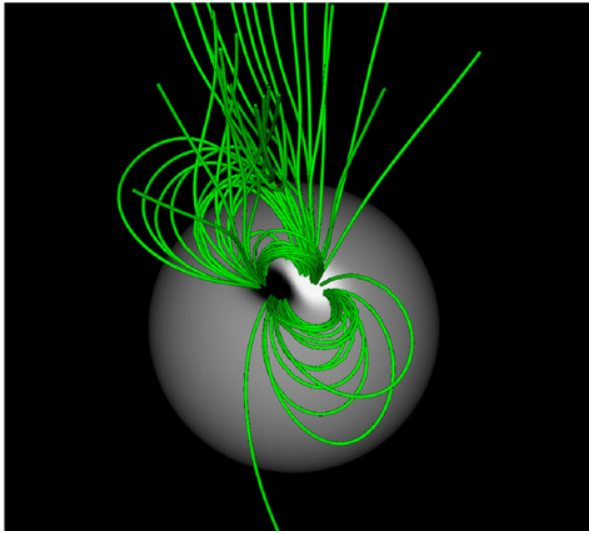

(i) Original, hour 696

Figure 6. Selected magnetic field lines illustrate the single bipole simulation at different times during the evolution. The original simulation (green) and the improvedreconstruction simulation (blue) quickly build up sheared field (first two rows) above the polarity inversion line of the bipole and then erupt. In the last row, the original field lines have almost settled down after the eruption, while the improved-reconstruction field lines have just started to settle. The inductive-only simulation (red) builds up the sheared field much more slowly, resulting in a much later eruption (not shown in this figure).

correlation staying above 0.9 over the entire simulation. In contrast, by the end of the inductive-only simulation, it has a correlation of 0.7 . The decrease of correlation over time in both cases is due to an accumulation and propagation over time of small deviations, owing to the "memory" of previous interactions in the MF model. The correlation values in Figure 9 were averaged over depth $r$. If we instead look at the correlation over $r$, then we observe that there is also a drop when we move higher up into the corona. This is due to the fact that while the field is line-tied near the photosphere where the boundary conditions are imposed, the field has more freedom further away from the fixed layer.

In addition, Figure 8 demonstrates how the diagnostic quantities discussed above change for the improved-reconstruction case when we vary the cadence between the input maps, i.e., the frequency of imposing new photospheric 


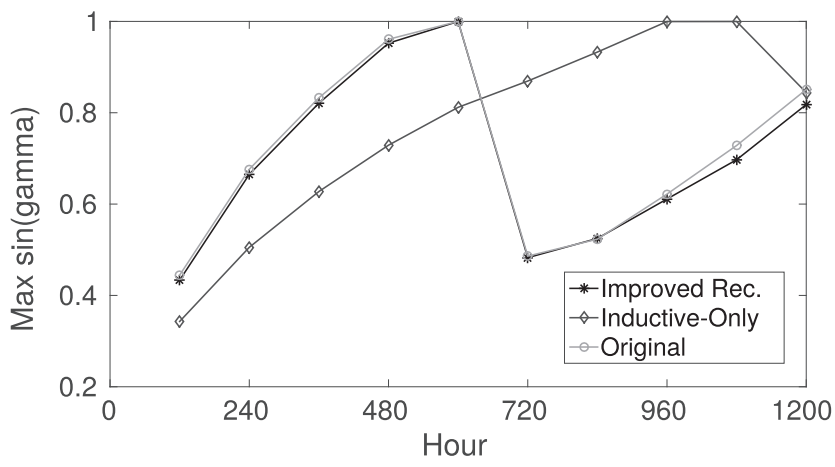

(a) Maximal skew at height $r=0.3448 R_{\odot}(\mathrm{z}=4)$ over time.

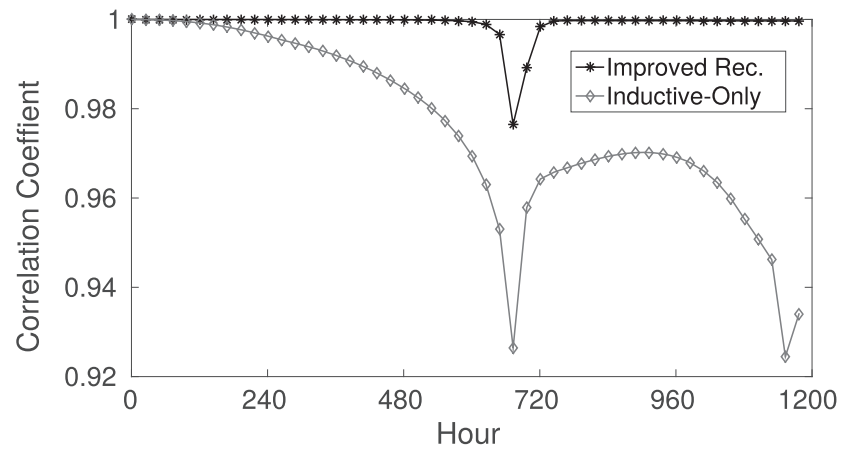

(b) Correlation of simulated $B_{\theta}$ (averaged in $r$ ) be-

tween each reconstruction and the original simula-

tion.

Figure 7. Comparison of different $\boldsymbol{E}_{\perp}$ reconstructions for the single bipole sequence.

boundary conditions. The curves are very close and always coincide at the input times. In between, the interpolation of the electric field produces a smooth curve. The effect of newly inserted bipoles is missed when the time step between new boundary conditions $d t>24 h$ (the cadence at which new bipoles emerged in the original simulation). This highlights the point that in any future application of this technique to the solar corona, it is very important that the cadence for the input of the maps be less than the cadence of introducing new emergences into the observed maps. Only through doing this will the full dynamics of the coronal field be captured.

Increasing the time step $d t$ (and thus decreasing the cadence) reduces the computational costs of the simulation slightly, as each time we impose a new electric field we have to solve two Poisson equations. This takes a few seconds on an average workstation using our multigrid Poisson solver (see Appendix A), but that adds up over the whole simulation run. However, one does not gain anything in the time-stepping process, which dominates the computational load. Note that the results in Section 5.3 indicate that the ADAPT maps are more sensitive to a change in $d t$.

We tested two alternative computational methods for imposing the photospheric boundary conditions: (i) interpolating the input maps $B_{r}$ and calculating a new electric field for each time step; and (ii) using the same electric field for the whole hour until a new electric field is imposed. (Our original method was to interpolate the electric field between input maps.) The first alternate method increases the computational workload immensely, whereas for the second method the gain in computational time is minimal. We did not find significant differences or improvements in the output with these methods.

\subsection{ADAPT Simulation}

Figure 10 shows the same quantities for the ADAPT simulation as seen previously for the single and multiple bipole cases. In the photospheric flux and (less distinctly) in the magnetic energy, one can see when new data are assimilated in the ADAPT data set once per day. Overall, the two curves of the improved and inductive-only cases are much closer than in the runs with the single and multiple bipole sequences. This is due to the presence of small-scale features in the ADAPT maps (see Figure 11), compared to the smooth average $B_{r}$ in the previous flux-transport model. The motion of these small-scale features allows the inductive electric field to better capture the advection of footpoints by differential rotation than in the bipole simulations, since the field at this location is no longer axisymmetric. In the bipole simulations, on the other hand, we have more large-scale features along the direction of the differential rotation (as in Figure 4). With a more or less constant $B_{r}$ along this flow direction, the motion is missed by the inductive component of the electric field. The higher values for the photospheric flux and the total current for the run with the ADAPT sequence are also due to the inclusion of smallscale features.

Although at first sight it might seem that the purely inductive reconstruction is sufficient for this sequence of $B_{r}$ maps, there are still times where the two simulations differ. This can be most easily seen in the open flux displayed in Figure 10 where, around hour 1000, more smooth large-scale features occur in the ADAPT maps. Therefore, the non-inductive component is still important to capture the evolution of these features. It will be interesting to consider in future work how the inductiveonly and the combined electric fields influence simulations over larger timescales.

One feature that catches the eye in the ADAPT tests is the large drop in energy around hour 352 in the simulation. Figure 11 demonstrates what happens in the simulation at that time: a large active region southern hemisphere (AR 12209, which was AR 12192 in the previous rotation) changes its structure while it is on the far side of the Sun. This change is recognized in the simulation when this region rotates into view. The large sudden change in the magnetic field results in a large electric field, due to Equation (6), which in turn influences the magnetic field, leading to a rapid rearrangement of magnetic field lines (discussed below). As we solve Poisson equations for the electric field, i.e., elliptic equations, the effect of this change is felt further into the domain, and creates the halos that we see in the middle column of Figure 11 (note the change of scale for the electric field components). After a short time, this defect is corrected by the simulation, as can be seen in the right-hand column of Figure 11. Similar halo effects can be seen when new bipoles are inserted in the multiple bipole simulation, and have been observed by M. D. Kazachenko et al. (2015, private communication). We are currently testing methods to remove the halos or at least reduce them.

As shown in Figure 12, during the assimilation of the new region, the free energy in the simulation increases. This 

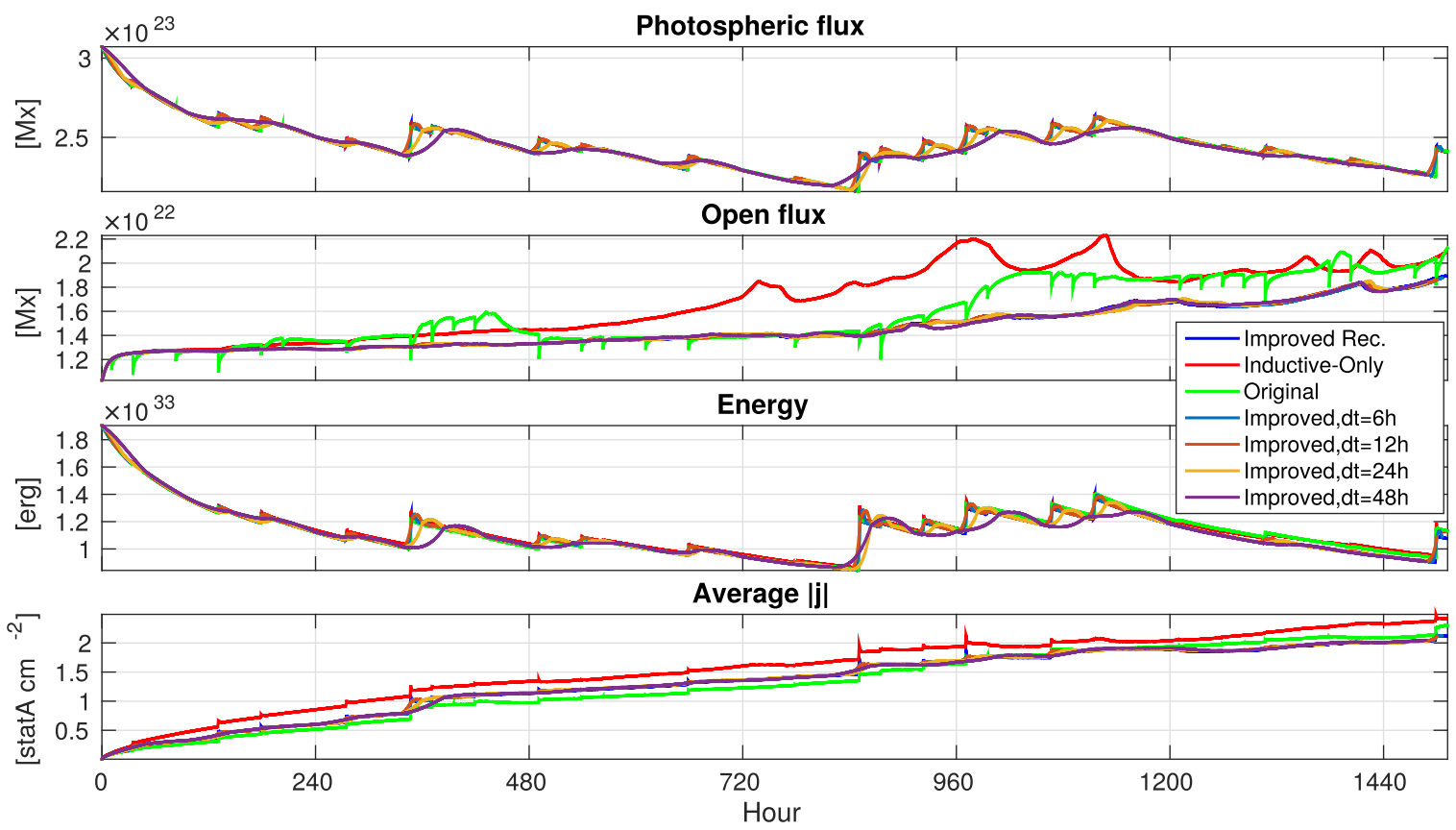

Figure 8. Same quantities as in Figure 5, but for the multiple bipole simulations, including the purely inductive reconstruction and a sequence of combined reconstructions with different time intervals $d t$ between input maps. The first three curves used $d t=1 h$.

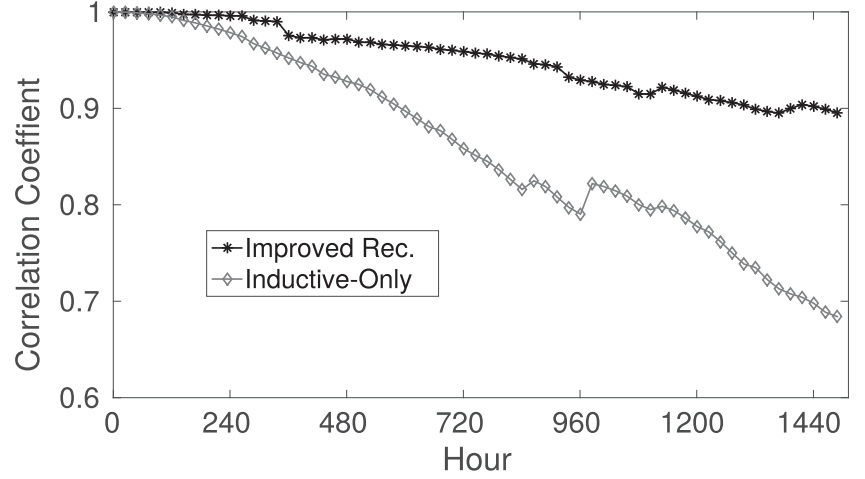

Figure 9. Correlation coefficient (averaged over $r$ ) of $B_{\theta}$ in the improvedreconstruction multiple bipole simulation and the inductive-only reconstruction multiple bipole simulation, respectively, with the original simulation.

happens just after the "big drop," when the potential field energy drops due to the drop in the photospheric flux, and is related to disconnection happening in the magnetic field, as described in the next paragraph. Afterward, it decays gradually back to a fairly constant value, but at a slightly higher level than before. This might be due to the assimilation in ADAPT and the corresponding electric field we chose. Future work might show to what extent the halo causes additional free energy outside the active region.

Looking at the magnetic field lines, we can observe that a large flux rope eruption takes place in our simulation shortly after AR 12209 rotates into view (see Figure 13), as was indicated by the peak in the open flux in Figure 10. In the MF model, this eruption was inevitable because the photospheric flux content of the region decreased substantially upon reassimilation, leaving disconnected magnetic field lines in the corona. Thus, when an eruption takes place, it is due to the rapid, near instantaneous change in the surface field due to the assimilation technique. It is not a consequence of the gradual process of building stress up in the coronal magnetic field by observed large-scale processes, as we have described before (Mackay \& van Ballegooijen 2006).

One rotation later, around hour 1012, the same active region reappears as AR 12237. A bit later, another hump in the open flux is visible, which is associated with another flux rope eruption. This later eruption is mainly due to a number of smaller bipoles which appear. Inspecting the observational data for AR 12192/12209, we find that in fact no eruptions took place at those times (as was to be expected). Instead, this region is known to have produced many, sometimes large flares, but only one associated CME during the previous rotation as AR 12192 on the Earth-side, which is quite unusual (see Sun et al. 2015; Thalmann et al. 2015). Since only near-side data were assimilated, we did not find evidence for or against a CME when the region was on the far side. As the flux rope eruptions in our simulation are associated with sudden rearrangement of the magnetic field lines due to newly assimilated data, a smoother transition for structural changes which appear at the far side might well change the nature or timing of associated eruptions in the simulation.

Compared to Figure 8, the variation of the time step size in Figure 10 for the improved-reconstruction ADAPT simulation seems to have a larger impact, especially on the open flux. The smoothing of the (unphysical) peaks in the photospheric flux at the data assimilation times for larger $d t$ may actually be beneficial. However, too large a value for $d t$ may lead to actual physical effects being missed if the photospheric maps are less smooth. Up to $d t=6 h$, the features of the curves still match each other pretty well, with just some smoothing added. However, for the two open flux peaks (around hour 352 and after hour 1000) the open flux curves for larger $d t$ are qualitatively different (in fact, $d t=8 h$, which is not shown here, also matches the $d t=2 h$ curve quite well until hour 1000, after which it becomes different). 

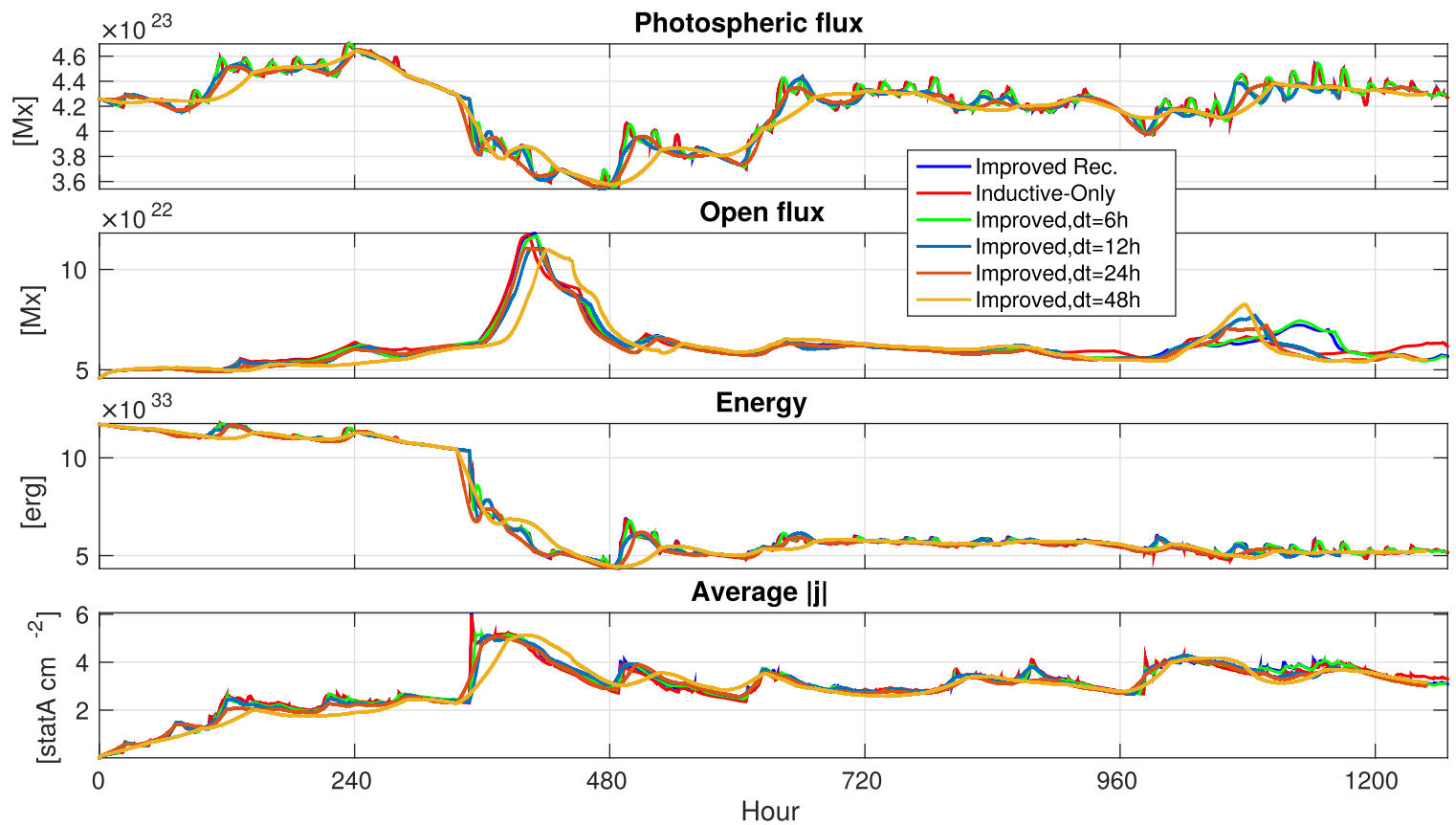

Figure 10. Same quantities as in Figure 8, but for the ADAPT-driven simulation. Runs with varying time $d t$ between input maps all use the improved reconstruction of $\boldsymbol{E}_{\perp}$. The first two curves used $d t=2 h$.

In Figures 14 and 15, we show the current-helicity density

$$
\alpha=\frac{\boldsymbol{B} \cdot \boldsymbol{j}}{B^{2}}=\frac{\boldsymbol{B} \cdot(\nabla \times \boldsymbol{B})}{B^{2}} .
$$

Since the coronal magnetic field is close to force-free in the MF model, this quantity essentially illustrates the locations of electric current and free energy. We only show the results for $d t \leqslant 24 h$. The two chosen times are as follow: (i) during the large flux rope eruption in the simulation following the appearance of AR 12209, and (ii) just before it comes around again as AR 12237. Interestingly, at this second time (hour 962), when all of the lines in Figure 8 match closely, the differences in $\alpha$ are quite large at some locations: in particular, between $d t=2$ and $d t=6$, at around latitude $50^{\circ}$. For hour 386 , on the other hand, $\alpha$ is rather similar for all of the shown $d t$ cases, despite the rather unpredictable behavior of the field lines during the eruption. A more quantitative difference in the value of $\alpha$ can be seen in Figure 15 where, for the two cases shown in Figure 14, the value of $\alpha$ averaged in longitude is shown as a function of latitude. Here, the main difference at $\theta=50^{\circ}$ for hour 962 (Figure 15(b)) is clear, where, as the time step varies from 2 to $24 \mathrm{hr}$, the value of $\alpha$ at this location changes significantly from positive to negative. In contrast, for hour 386 (Figure 15(a)), the sign of $\alpha$ at a given latitude is the same no matter what cadence is used.

In light of the discussion from Section 5.2 concerning the limited gain in computational time, the main issue concerning the choice of $d t$ remains the availability of the $B_{r}$ maps, which are mostly available at $24 \mathrm{hr}$ intervals. We should therefore keep in mind that this has a non-negligible influence on the simulation. One consequence of this is that in the future, wherever possible, a variety of cadences should be used along with ensemble averaging.
We already mentioned in Section 3.3 that the ADAPT data consist of an ensemble of 12 different realizations from which we deliberately picked realization 1 for our simulation. In Figure 16, the same diagnostic quantities considered before are compared for the members of the ensemble. The curves match very closely, except from the open flux, which is, as we have seen before, the most sensitive quantity. Future longer simulation runs might show whether or not the choice of the realization has a long-term significant influence on the simulation output.

\section{CONCLUSION}

We have demonstrated how a global non-potential coronal magnetic field simulation using the magnetofrictional method can be successfully driven by a time sequence of radial magnetic field maps on the photosphere. Two possible solutions for the electric field reconstruction have been considered: a purely inductive solution and one including a non-inductive correction. The second solution was motivated by tests with our existing flux-transport model, where it lead to a substantial improvement in the reconstruction of the (known) electric field. The correction accounts for the non-inductive electric field caused by the large-scale differential rotation. We have also applied both electric fields to a sequence of maps from the ADAPT model where the true electric field is not known (due to the assimilation of observed data). In this case, we find that correcting for the non-inductive contribution of differential rotation is less important, although it does have some effect later in the simulation.

By varying parameters for the reconstruction of the photospheric electric field in the simulation, we found that the time step between the input maps has an effect that can become important at some points. For a time step of up to $d t=6 h$, we found the results to be reasonably close to the $d t=2 h$ simulation, but for larger $d t$, differences such as earlier flux 

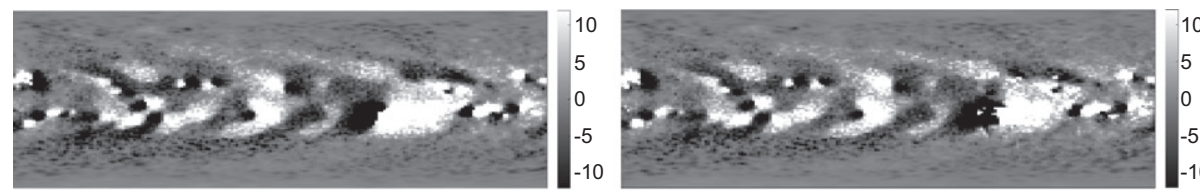

(a) $B_{r} 320$ hours into the simulation.
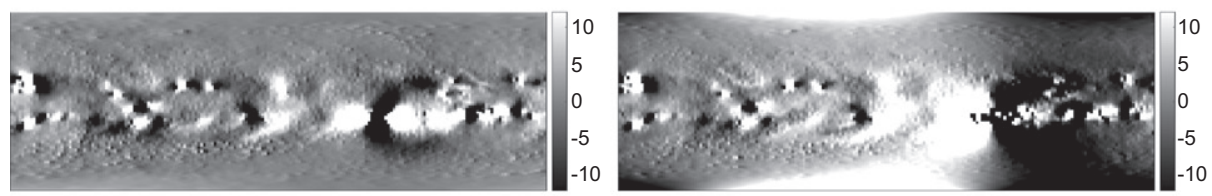

(d) $B_{\phi} 320$ hours into the simulation.
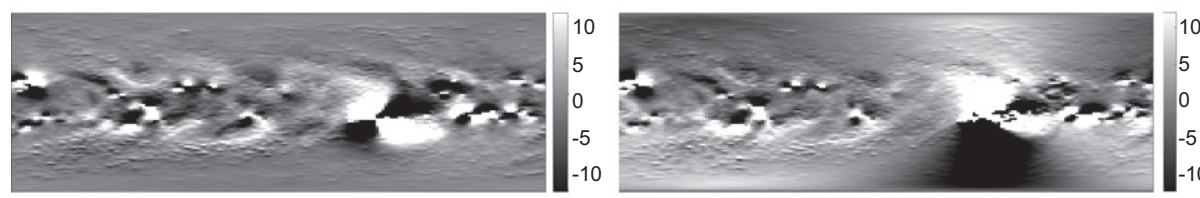

(g) $B_{\theta} 320$ hours into the simulation.
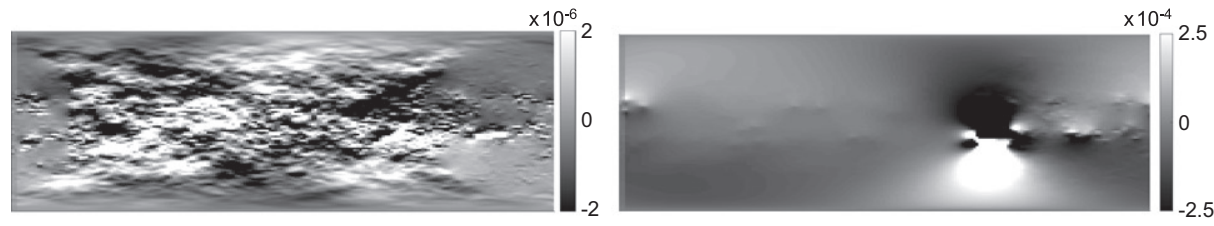

(k) $E_{\phi} 352$ hours into the simulation.

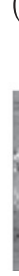

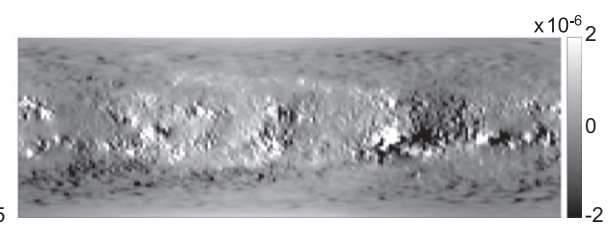

(o) $E_{\theta} 370$ hours into the simulation.

(m) $E_{\theta} 320$ hours into the simulation.

(n) $E_{\theta} 352$ hours into the simulation.

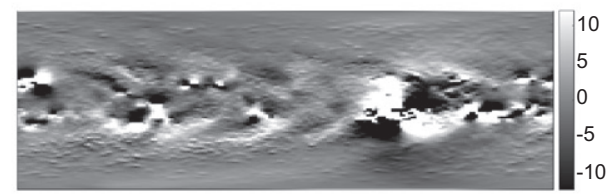

(i) $B_{\theta} 370$ hours into the simulation.

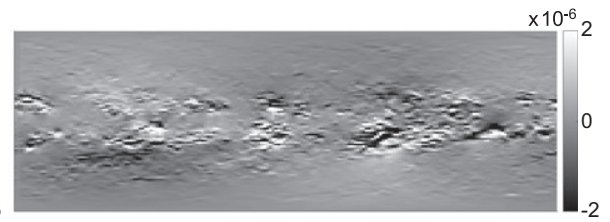

(1) $E_{\phi} 370$ hours into the simulation.

Figure 11. Magnetic and electric fields during the big energy drop (assimilation of AR 12209, which is the large active region in the bottom right quadrant of each field map, clearly visible in the top row) in the combined-reconstruction ADAPT simulation. Note the different scale for the electric field in the middle column.

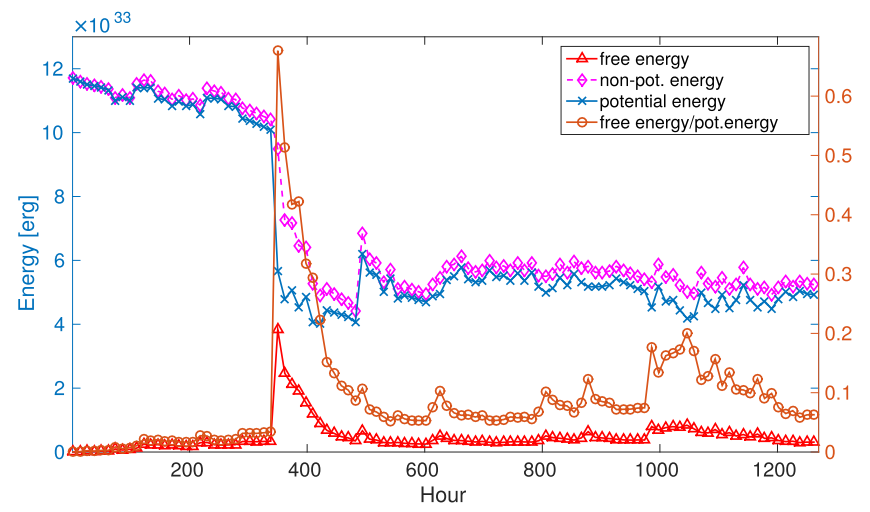

Figure 12. Evolution of the potential energy, non-potential energy, free energy, and relative energy (free/potential energy, note the different $y$-axis) in the improved-reconstruction ADAPT simulation. rope eruptions start to appear. In between the data input times, it is sufficient to just interpolate the resulting electric field linearly.

There remain some limitations to our approach, which we are currently working on. For example, large and sudden changes in the photospheric field-for example when a new bipole appears or an active region that has changed its structure on the far side is assimilated-lead to a large short-term increase of the electric field. This in turn can have a significant effect on the evolution of the coronal field, such as the initiation of an eruption. Due to the nature of our reconstruction, this change is felt outside the direct region of change in the photospheric magnetic map, potentially influencing the largerscale free energy and open flux. It will therefore be desirable, in the future, to find an approach to electric field reconstruction (i.e., a choice of non-inductive component) that minimizes 


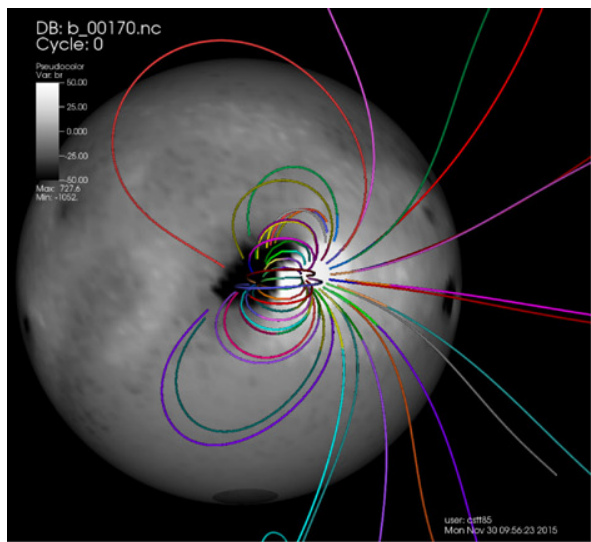

(a) Hour 340

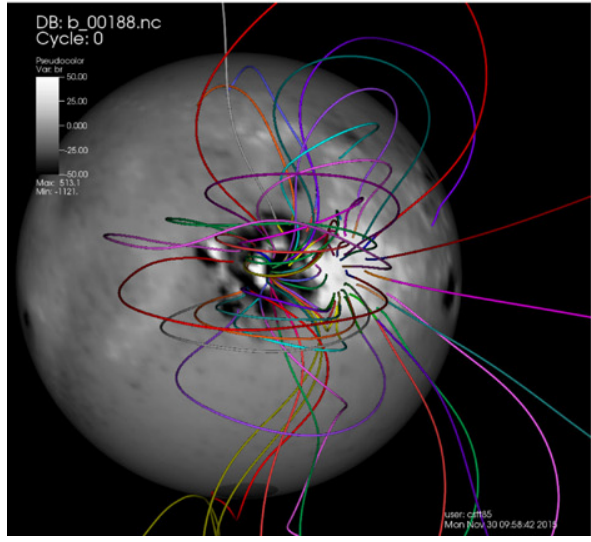

(d) Hour 376

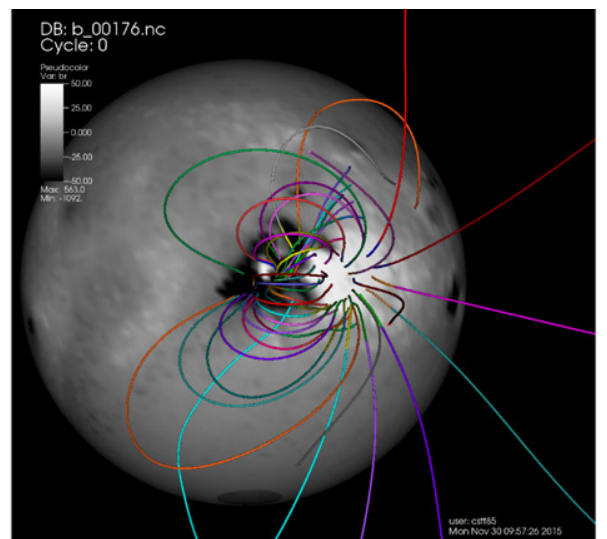

(b) Hour 352

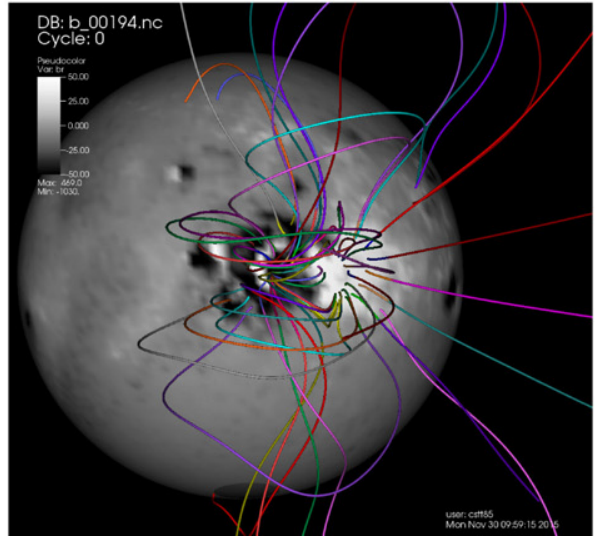

(e) Hour 388

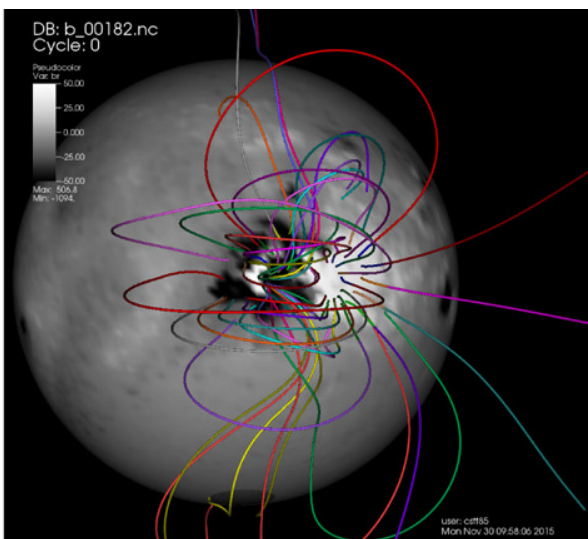

(c) Hour 364

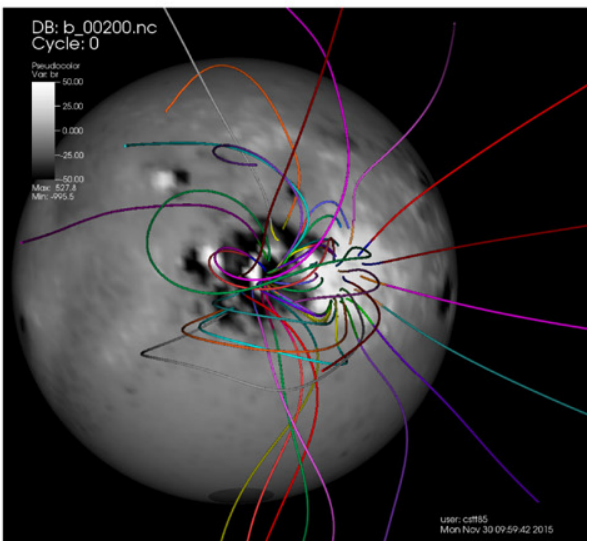

(f) Hour 400

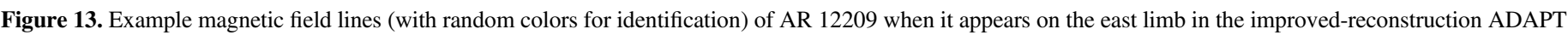
simulation. Due to the structural change in the active region, the magnetic field lines rearrange and erupt.

these so-called halos. Alternatively, improved treatment of the assimilation discontinuity when constructing the ADAPT maps themselves may lead to improved simulation results for this data set. Recent work by Arge et al. (2013) describes how additional helioseismic far-side detection techniques can be used to detect new emerging, large active regions on the farside of the Sun and to assimilate these before they arrive at the east limb. Hickmann et al. (2015) describe further improvements to the ADAPT model, in particular, by using local ensemble transform Kalman filtering instead of ensemble least squares. Using this improved data when it becomes available might reduce the effect of the unrealistic sudden changes in the photospheric field.

We have observed two different processes that can lead to flux rope ejection: (i) a slow, gradual build up of stress in the magnetic field, as described by Mackay \& van Ballegooijen (2006); and (ii) rapid changes in the electric field, which are, in our case, partly due to the data assimilation technique in the ADAPT maps.

A further contribution to the non-inductive electric field which we have not yet included is that of additional magnetic helicity injection. This may arise either from the large-scale twisted fields in newly emerging active regions (Yeates et al. 2007, 2008), or from the cumulative effect of smallscale vortical motions in the photosphere (Antiochos 2013; Mackay et al. 2014; Knizhnik et al. 2015). Both forms of helicity injection have been shown to influence the amount and distribution of free magnetic energy in the corona, however, neither form is fully accounted for in global radial-field maps. Even the inductive part of this contribution (due to the horizontal advection of $B_{r}$, for example) may be missed in current maps because the growth of a particular active region may not be followed while it is on the far side of the Sun.

Nevertheless, we have demonstrated that magnetofrictional models of the corona may be successfully driven from sequences of photospheric magnetic maps. Our approximation to the non-inductive electric field due to differential rotation may also be useful in MHD simulations. In the future, this new technique may be applied for space weather models that use a combination of observations and theoretical modeling to simulate the variation of the Sun's open flux and flux rope ejections.

This work is supported by a grant from the US Air Force Office of Scientific Research (AFOSR) in the AFOSR Basic Research Initiative "Understanding the Interaction of CMEs with the Solar-Terrestrial Environment." D.H.M. would like to thank the Leverhulme Trust and the UK STFC for financial support.

The work utilizes data produced collaboratively between Air Force Research Laboratory (AFRL) and the National Solar Observatory (NSO). The ADAPT model development is 


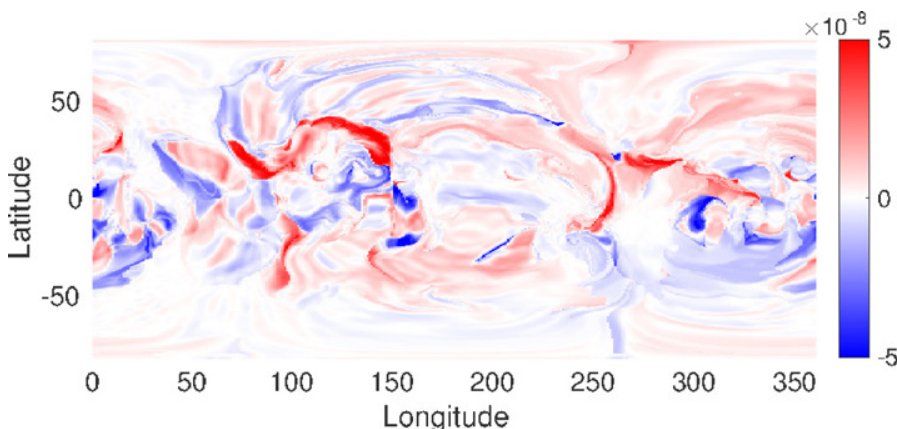

(a) Hour $386, d t=2 h$

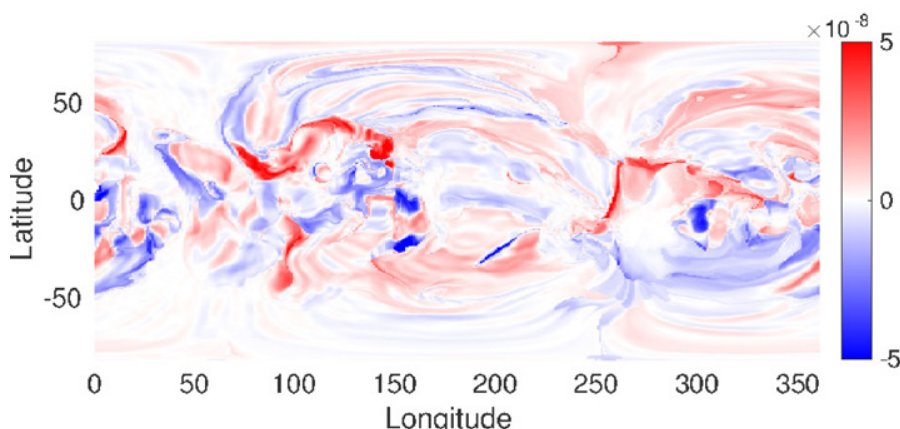

(c) Hour 386, $d t=12 \mathrm{~h}$

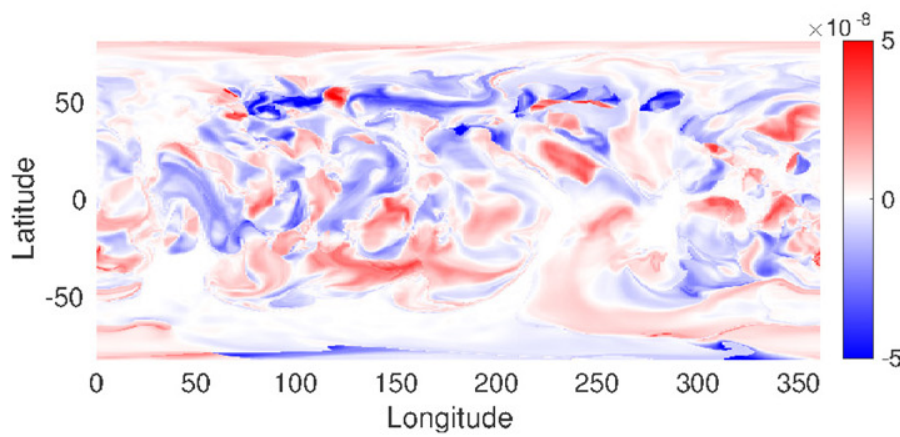

(e) Hour 962, $d t=2 h$

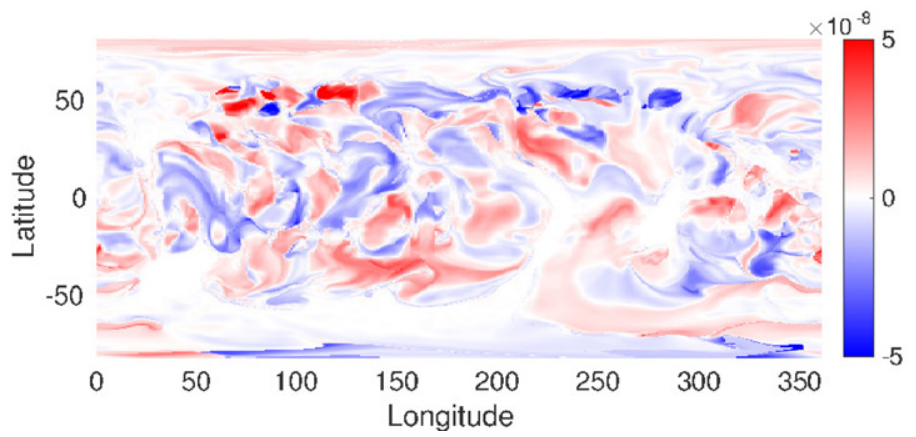

(g) Hour 962, $d t=12 h$

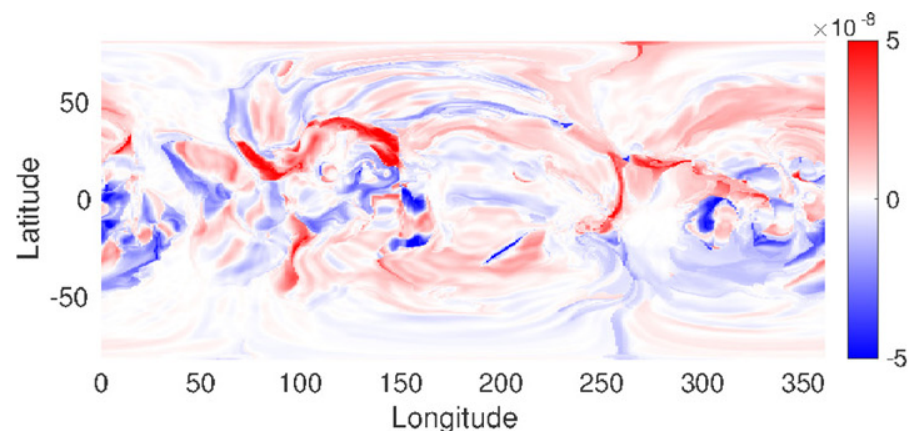

(b) Hour 386, $d t=6 h$

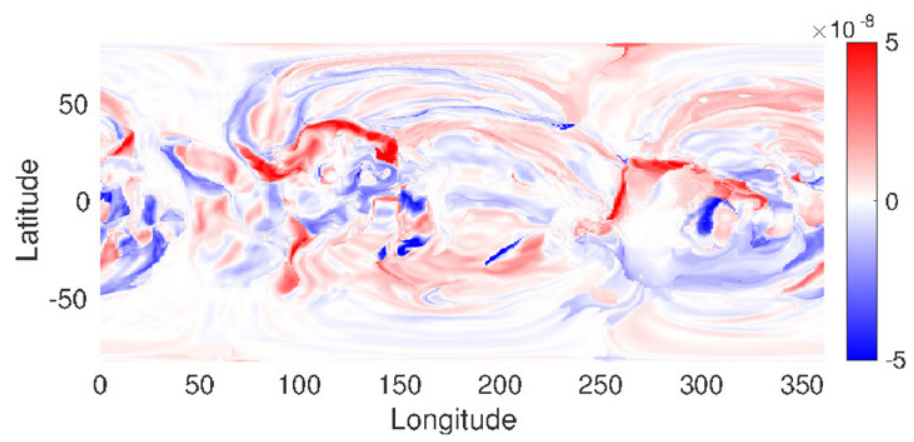

(d) Hour 386, $d t=24 h$

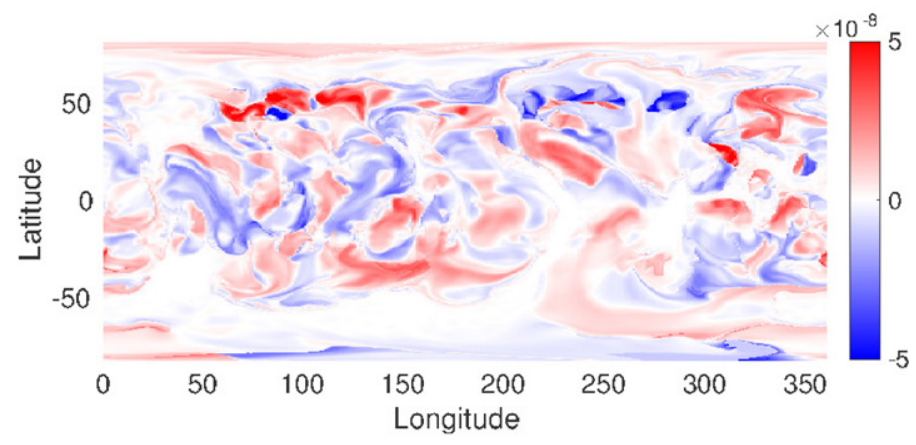

(f) Hour 962, $d t=6 h$

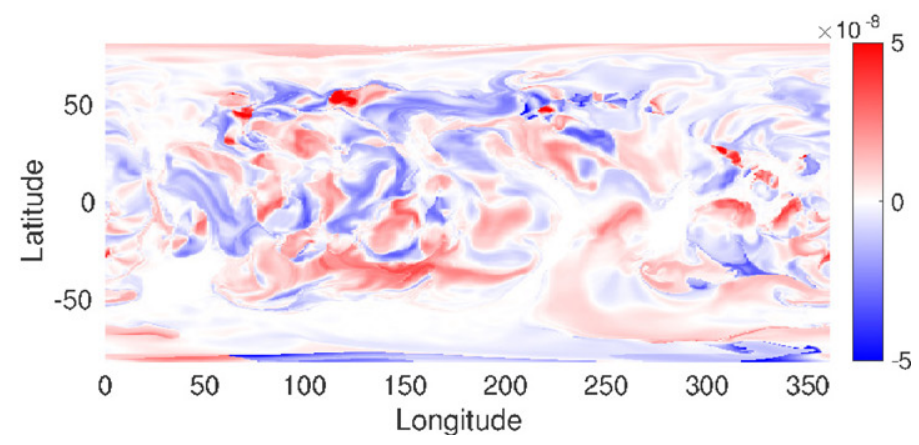

(h) Hour 962, $d t=24 h$

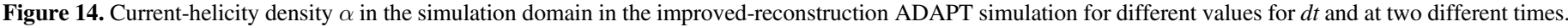
Here, $\alpha$ has been averaged along magnetic field lines and is plotted on a sphere at height $r=1.03327$. Units are $\mathrm{m}^{-1}$.

supported by AFRL and AFOSR. The input data utilized by ADAPT are obtained by NSO/NISP (NSO Integrated Synoptic Program). NSO is operated by the Association of Universities for Research in Astronomy (AURA), Inc., under a cooperative agreement with the National Science Foundation (NSF).

We thank Xudong Sun for useful suggestions.
APPENDIX A

\section{NUMERICAL METHODS FOR POISSON EQUATIONS}

\section{A.1. Computational Coordinates}

So far, we have used standard spherical coordinates notation to define the equations to be solved in the simulation. Our 


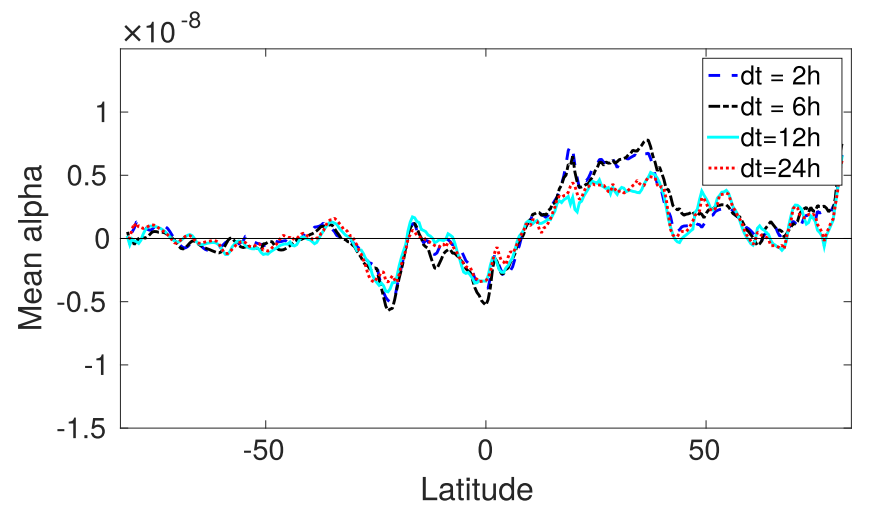

(a) Hour 386

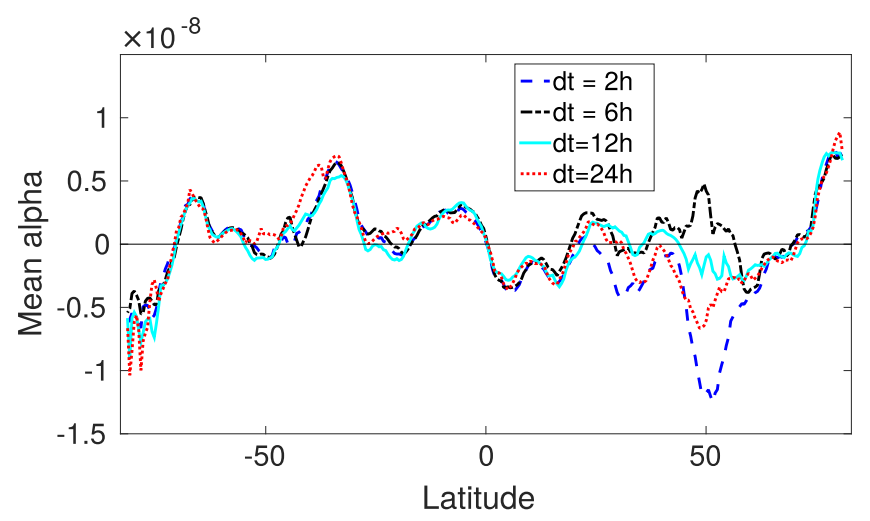

(b) Hour 962

Figure 15. Mean $\alpha$ (i.e., current-helicity density $\alpha$ averaged over longitude) at height $r=1.03327$ for different values of $d t$ and at the same two times as in Figure 14.

computational grid, however, is curvilinear, and the computational coordinates $(x, y, z)$ are defined in terms of $(\phi, \theta, r)$, as in van Ballegooijen et al. (2000), by

$$
\begin{aligned}
x & =\frac{\phi}{\Delta_{\phi}}, \\
y & =-\frac{\log (\tan (\theta / 2))}{\Delta_{\phi}} \\
\text { and } \quad z & =\frac{\log \left(r / R_{\odot}\right)}{\Delta_{\phi}},
\end{aligned}
$$

where $\Delta_{\Phi}$ is the azimuthal cell size (in radians) at the equator. The scale factors are given by $h_{x}=h_{y}=\Delta_{\Phi} r \sin (\theta)$ and $h_{z}=\Delta_{r}$. This grid is uniformly spaced in $x, y$, and $z$, with cell sizes increasing linearly with radius. Since we remain on the surface of the sphere when solving the Poisson Equations (8) and (10), we only need a two-dimensional (2D) Poisson solver. In Appendix A.2, we describe the solver we use in our simulation.

The poles are cut off at $\pm 81.7^{\circ}$ in order to avoid having to deal with the pole problem (see, e.g., Ronchi et al. 1996; Kageyama \& Sato 2004), and to avoid time-stepping restrictions due to the convergence of the cell sizes toward the poles. See Yeates (2014) for a possible solution by using a variable grid that doubles the cell size toward the pole. We plan to integrate this into our simulation code.
Instead of solving the equations on the curvilinear grid defined by the above coordinates, we take a closer look at the stencil we receive from a finite volume discretization of Equation (8) in curvilinear coordinates. With $h_{e x}$ and $h_{e y}$ being the distances of the cell centers in the horizontal and vertical directions, and using compass notation, we obtain

$$
B_{z}(x, y)=-\frac{1}{h_{x} h_{y}}\left[\begin{array}{ccc}
0 & \frac{h_{e x}^{N}}{h_{e y}^{N}} & 0 \\
\frac{h_{e y}^{W}}{h_{e x}^{W}} & -\Sigma & \frac{h_{e y}^{E}}{h_{e x}^{E}} \\
0 & \frac{h_{e x}^{S}}{h_{e y}^{S}} & 0
\end{array}\right] \Phi(x, y),
$$

with $\Sigma=h_{e x}^{N} / h_{e y}^{N}+h_{e y}^{E} / h_{e x}^{E}+h_{e x}^{S} / h_{e y}^{S}+h_{e x}^{W} / h_{e y}^{W}$. Due to the uniform spacing, we can assume $h_{e x}^{D}=h_{e y}^{D}$ for $D \in\{N, E, S, W\}$, and therefore we obtain the standard Laplace five-point stencil:

$$
B_{z}=-\frac{1}{h_{x} h_{y}}\left[\begin{array}{rrr}
0 & 1 & 0 \\
1 & -4 & 1 \\
0 & 1 & 0
\end{array}\right] \Phi(x, y) .
$$

By multiplying both sides of the equation by $h_{x} h_{y}$, the stencil becomes that for the Cartesian grid. This means that by multiplying the right-hand side of the Poisson equation by $h_{x} h_{y}=\Delta_{\Phi}^{2} \sin ^{2}(\theta)$, we can obtain $\Phi$ by solving the equation on a Cartesian grid.

Similarly, the discretized form of Equation (10) in curvilinear coordinates can be solved on a Cartesian grid. In the next section, the benefit of doing so is demonstrated.

\section{A.2. Poisson Solver}

We have implemented a geometric multigrid solver (see, e.g., Briggs et al. 2000; Trottenberg et al. 2001 and references therein) which uses Red-Black Gauss-Seidel smoothing, weighted averaging as restriction, and prolongation based on the Taylor expansion (in order to preserve magnetic flux; see, e.g., van der Holst \& Keppens 2007). Our simulation grid in this work is a $192 \times 160$ cell-centered grid which we coarsen down to $12 \times 10$, which means that we have five levels in the multigrid hierarchy. Horizontally, we apply periodic boundary conditions. On the top and bottom, we have homogenous Neumann boundary conditions for solving Equation (8) and homogenous Dirichlet for Equation (10). We perform $\mathrm{F}(2,1)$ cycles, i.e., two pre- and one postsmoothing step. F cycles, as defined in Brandt (1984), are about as effective as W cycles, but they are less computationally expensive. The iteration is stopped when the residual $r_{i}=f-A u_{i}$ (with subscript $i$ the iteration number, $f$ the right-hand side of the equation, $A$ the system matrix, and $u_{i}$ the current approximation to the solution) is smaller than or equal to $1.0 \times 10^{-8}$.

To analyze the efficiency of the solver, we take a look at the convergence factor $\rho=r_{i+1} / r_{i}$, and its geometric mean $\rho_{\text {mean }}=\sqrt[n]{r_{\text {end }} / r_{\text {start }}}$, and we also consider $\mathrm{V}(2,1)$ cycles. Table 1 shows how the solver performs for the multiple bipole simulation, both on the original curvilinear and on the Cartesian grid discussed in Appendix A.1. Results are shown both for the Neumann problem (inductive part of the electric field) and for the Dirichlet problem (non-inductive part), using 

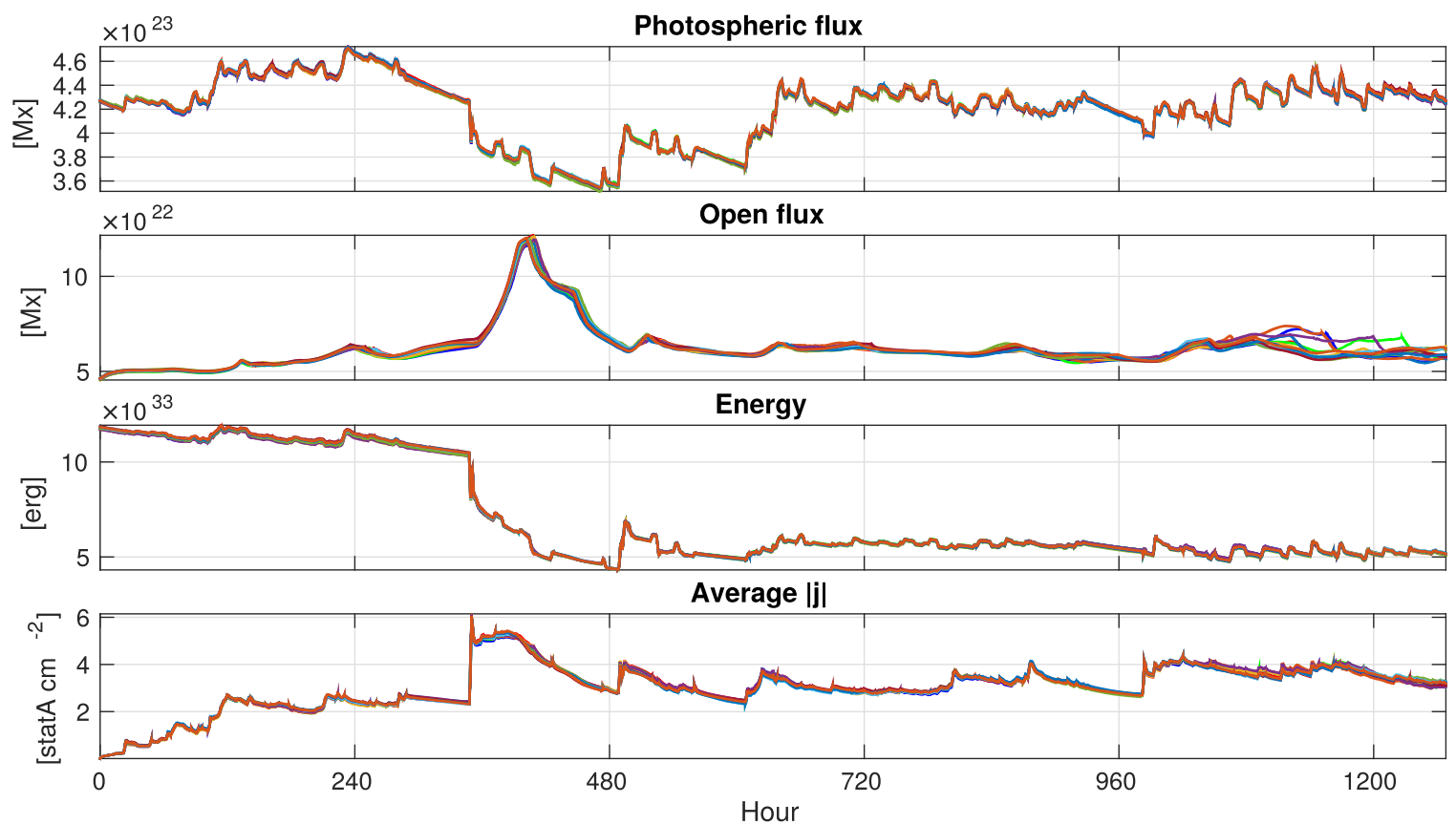

Figure 16. Same quantities as in Figure 8, but for the 12 different members of the ADAPT ensemble (shown in different colors) in the improved-reconstruction case. All runs used $d t=2 h$.

Table 1

Convergence of Multigrid Poisson Solver

\begin{tabular}{lccccc}
\hline \hline & \multicolumn{2}{c}{ Curvilinear } & & \multicolumn{2}{c}{ Cartesian } \\
\cline { 2 - 3 } \cline { 5 - 6 } Cycle/BC & $\rho_{\text {mean }}$ & \#Iterations & & $\rho_{\text {mean }}$ & \#Iterations \\
\hline $\mathrm{V}(2,1) / \mathrm{NM}$ & $*$ & $*$ & & 0.44 & 30 \\
$\mathrm{~F}(2,1) / \mathrm{NM}$ & $*$ & $*$ & & 0.24 & 17 \\
$\mathrm{~V}(2,1) / \mathrm{DIR}$ & 0.34 & 31 & & 0.25 & 18 \\
$\mathrm{~F}(2,1) / \mathrm{DIR}$ & 0.19 & 18 & & 0.14 & 13 \\
\hline
\end{tabular}

Note. Convergence behavior of the multigrid Poisson solver for stopping criterion $r_{n} \leqslant 1.0 \times 10^{-8}$, Neumann (NM) or Dirichlet (DIR) top and bottom boundaries, and $\mathrm{V}$ or $\mathrm{F}$ cycles. An asterix refers to a stalled iteration $\left(\rho_{\text {end }} \approx 1\right)$ before the stopping criterion was reached.

the stopping criterion imposed in our simulation. One can observe that the cell-centered grid, combined with Neumann boundaries, poses some challenges to the solver on the curvilinear grid where the residual stalls at a certain level due to boundary effects. On a Cartesian grid, however, the solver shows reasonable performance for all of the setups. For the setup in our simulation, we reach the criterion after 17 iterations with a mean convergence factor of 0.24 in the Neumann case, and $\rho_{\text {mean }}=0.14$ after 13 iterations in the Dirichlet case. In tests with different stopping criteria, we observed that up to a relative stopping criterion of $r_{n} / r_{\text {start }} \leqslant 1.0 \times 10^{-6}$ the solver also converged on the curvilinear grid with Neumann boundaries.

\section{REFERENCES}

Amari, T., Luciani, J. F., Aly, J. J., Mikic, Z., \& Linker, J. 2003, ApJ, 595,1231

Antiochos, S. K. 2013, ApJ, 772, 72

Arge, C. N., Henney, C. J., Hernandez, I. G., et al. 2013, AIPC, 1539, 11

Arge, C. N., Henney, C. J., Koller, J., et al. 2010, AIPC, 1216, 343

Brandt, A. 1984, Multigrid Techniques: 1984 Guide with Applications to Fluid Dynamics, GMD-Studien No. 85 (St. Augustin, Germany: GMD-AIW)
Briggs, W. L., Henson, V. E., \& McCormick, S. F. 2000, A Multigrid Tutorial (2nd ed.; Philadelphia: SIAM)

Cheung, M. C. M., \& DeRosa, M. L. 2012, ApJ, 757, 147

Edwards, S., Yeates, A., Bocquet, F.-X., \& Mackay, D. 2015, SoPh, 290, 2791

Fisher, G., Welsch, B., \& Abbett, W. 2012, SoPh, 277, 153

Fisher, G. H., Welsch, B. T., Abbett, W. P., \& Bercik, D. J. 2010, ApJ, 715,242

Gibb, G. P. S., Mackay, D. H., Green, L. M., \& Meyer, K. A. 2014, ApJ, 782,71

Harvey, J. W., Hill, F., Hubbard, R. P., et al. 1996, Sci, 272, 1284

Henney, C. J., Toussaint, W. A., White, S. M., \& Arge, C. N. 2012, SpWea, 10, S02011

Hickmann, K. S., Godinez, H. C., Henney, C. J., \& Arge, C. N. 2015, SoPh, 290, 1105

Kageyama, A., \& Sato, T. 2004, GGG, 5, Q09004

Kazachenko, M. D., Fisher, G. H., \& Welsch, B. T. 2014, ApJ, 795, 17

Knizhnik, K. J., Antiochos, S. K., \& DeVore, C. R. 2015, ApJ, 809, 137

Mackay, D., \& Yeates, A. 2012, LRSP, 9, 6

Mackay, D. H. 2006, ApJ, 642, 1193

Mackay, D. H., DeVore, C. R., \& Antiochos, S. K. 2014, ApJ, 784, 164

Mackay, D. H., Green, L. M., \& van Ballegooijen, A. A. 2011, ApJ, 729, 97

Mackay, D. H., \& van Ballegooijen, A. A. 2001, ApJ, 560, 445

Mackay, D. H., \& van Ballegooijen, A. A. 2005, ApJL, 621, L77

Mackay, D. H., \& van Ballegooijen, A. A. 2006, ApJ, 641, 577

Ronchi, C., Iacono, R., \& Paolucci, P. S. 1996, JCoPh, 124, 93

Schrijver, C. J., \& DeRosa, M. L. 2003, SoPh, 212, 165

Schüssler, M., \& Baumann, I. 2006, A\&A, 459, 945

Snodgrass, H. B. 1983, ApJ, 270, 288

Sun, X., Bobra, M. G., Hoeksema, J. T., et al. 2015, ApJL, 804, L28

Thalmann, J. K., Su, Y., Temmer, M., \& Veronig, A. M. 2015, ApJL, 801, L23

Trottenberg, U., Oosterlee, C. W., \& Schüller, A. 2001, Multigrid (San Diego: Academic)

Upton, L., \& Hathaway, D. H. 2014, ApJ, 780, 5

van Ballegooijen, A. A., Priest, E. R., \& Mackay, D. H. 2000, ApJ, 539, 983

van der Holst, B., \& Keppens, R. 2007, JCoPh, 226, 925

Worden, J., \& Harvey, J. 2000, SoPh, 195, 247

Yang, L. P., Feng, X. S., Xiang, C. Q., et al. 2012, JGRA, 117, A08110

Yang, W. H., Sturrock, P. A., \& Antiochos, S. K. 1986, ApJ, 309, 383

Yeates, A. R. 2014, SoPh, 289, 631

Yeates, A. R., \& Mackay, D. H. 2012, ApJL, 753, L34

Yeates, A. R., Mackay, D. H., \& van Ballegooijen, A. A. 2007, SoPh, 245, 87

Yeates, A. R., Mackay, D. H., \& van Ballegooijen, A. A. 2008, SoPh, 247, 103

Yeates, A. R., Mackay, D. H., van Ballegooijen, A. A., \& Constable, J. A. 2010, JGRA, 115, 9112 